\title{
Optimal Fuzzy Controller Design: Local Concept Approach
}

\author{
Shinq-Jen Wu and Chin-Teng Lin, Senior Member, IEEE
}

\begin{abstract}
In this paper, we present a global optimal and stable fuzzy controller design method for both continuous- and discrete-time fuzzy systems under both finite and infinite horizons. First, a sufficient condition is proposed which indicates that the global optimal effect can be achieved by the fuzzily combined local optimal controllers. Based on this sufficient condition, we derive a local concept approach to designing the optimal fuzzy controller by applying traditional linear optimal control theory. The stability of the entire closed-loop continuous fuzzy system can be ensured by the designed optimal fuzzy controller. The optimal feedback continuous fuzzy system can not only be guaranteed to be exponentially stable, but also be stabilized to any desired degree. Also, the total energy of system output is absolutely finite. Moreover, the resultant feedback continuous fuzzy system possesses an infinite gain margin; that is, its stability is guaranteed no matter how large the feedback gain becomes. Two examples are given to illustrate the proposed optimal fuzzy controller design approach and to demonstrate the proved stability properties.
\end{abstract}

Index Terms-Converse theorem, degree of stability, exponentially stable, finite energy, gain margin, global optimal, Riccati equation, T-S type fuzzy model.

\section{INTRODUCTION}

$\mathbf{N}$ ONLINEARITY and uncertainty are always bothersome in controlling a real system, since a physical system is usually partly known and difficult to describe, has few measurements available, or is highly nonlinear. Fuzzy modeling can mimic a real system well, fuzzy control can support more robust control than linear control does, and, moreover, optimal control can provide the best possible system. Hence, an analytic design scheme of the optimal fuzzy controller for a fuzzy system (i.e., the system described by a fuzzy model) is of theoretical and practical interest. Although the research in fuzzy modeling and fuzzy control has been quite matured [1]-[11], it seems that the field of optimal fuzzy control is nearly open [12]. The goal of this work is to propose a scheme for designing a global optimal fuzzy controller to control and stabilize a continuous- or discrete-time fuzzy system in finite or infinite horizon (time) consideration. A simple stability criterion is proposed and the gain margin of the resultant closed-loop fuzzy system is discussed.

Stability and optimality are the most important requirements for any control system. Most of the existed works are based on Takagi-Sugeno (T-S) type fuzzy model combined with parallel distribution compensation (PDC) concept [1] and apply Lyapunov's method to do stability analysis. Tanaka and coworkers

Manuscript received January 11, 1999; revised December 10, 1999. The authors are with the Department of Electrical and Control Engineering, National Chiao-Tung University, Hsinchu, Taiwan, R.O.C.

Publisher Item Identifier S 1063-6706(00)03204-5. reduced the stability analysis and control design problems to linear matrix inequality (LMI) problems [2], [4]. Furthermore, they relaxed the stability condition, and then, derived a fuzzy controller based on the relaxed LMI stability condition [13], [14]. Moreover, they also dealt with uncertainty issue [3]. This approach had been applied to several control problems such as control of chaos [4], of an articulated vehicle [5], of a mobile robot with multiple trailers [7], and of a modal car [15]. A frequency shaping method to achieve systematic design of fuzzy controllers was also performed by them [16]. Sun and coworkers developed a separation scheme to design a fuzzy observer and a fuzzy controller independently [9]. Methods based on grid-point approach [17] and circle criteria [18], [19] were introduced to do stability analysis of fuzzy control as well. Wang adopted a supervisory controller and introduced stability and robustness measures [20]. Cao proposed a decomposition principle to design a fuzzy discrete-time control system and an equivalent principle to do stability analysis [11]. Even with the aforementioned research results on the theoretic aspect of fuzzy control, Tanaka and others' work mentioned in the above always treat the stability of general linear feedback fuzzy controllers.

On the issue of optimal fuzzy control, Wang developed an optimal controller to stabilize a linear time-invariant system via Pontryagin maximum principle [12]. However, although fuzzy control of linear systems could be a good starting point for better understanding of some issues in fuzzy control synthesis, it does not have much practical implications since using the fuzzy controller designed for a linear system directly as the controller may not be a good choice [12]. Moreover, the cited stability criteria may be simple, but rough to do systematic analysis and also may result in a controller with less flexibility. Tanaka and coworkers [21], [22] tried to obtain a fuzzy controller to minimize the upper bound of the quadratic performance function by linear-matrix-inequality (LMI) approach based on the assumption of local-linear-feedback-gain control structure. Nevertheless, no theoretical analysis on this design scheme of optimal-fuzzy-control structure was proposed.

In this work, a global optimal fuzzy controller design method for a fuzzy system is achieved from a local viewpoint and the properties of the constructed optimal fuzzy controller are exposed based on the linear optimal control theory. The derived control law is demonstrated to be the best for the entire system to reach the optimal performance index. Moreover, the optimal feedback continuous fuzzy system can not only be guaranteed to be exponentially stable, but also be stabilized to any desired degree. Furthermore, we elicit that this kind of fuzzy controller can stabilize a continuous fuzzy system to any prescribed degree of stability, and the corresponding closed-loop continuous 
fuzzy system possesses an infinite gain margin. Moreover, the total energy of the system output of the feedback continuous fuzzy system is absolutely finite.

This paper is organized as follows. The sufficient condition of global optimum is proposed in Section II, which indicates that fuzzily "blending" the local optimal fuzzy controllers can achieve global optimal effect. The global optimal fuzzy control laws for both continuous- and discrete-time fuzzy systems during both finite and infinite horizons are derived theoretically in Section III. Several properties such as stability criteria and gain margin of the resultant closed-loop fuzzy system are discussed in Section IV. The design methodology is illustrated by two examples in Section V. Section VI gives the concluding remarks. The related linear optimal theory applied in this paper is summarized in the Appendix

\section{SyStem REPRESENTATION AND PROBLEM StATEMENT}

We consider a given nonlinear plant described by the so-called T-S type fuzzy model

$$
\begin{gathered}
R^{i}: \text { If } x_{1} \text { is } T_{1 i}, \cdots, x_{n} \text { is } T_{n i} \\
\text { then } S X(t)=A_{i}(t) X(t)+B_{i}(t) u(t) \quad i=1, \cdots, r \\
Y(t)=C(t) X(t),
\end{gathered}
$$

where $R^{i}$ denotes the $i$ th rule of the fuzzy model; $x_{1}, \cdots, x_{n}$ are system states; $T_{1 i}, \cdots, T_{n i}$ are the input fuzzy terms in the $i$ th rule; $S X(t)$ denotes $\dot{X}(t)$ for continuous case and $X(t+1)$ for discrete case; the state vector $X(t)=\left[x_{1}, \cdots, x_{n}\right]^{T} \in \Re^{n}$, the system output vector $Y(t) \in \Re^{n^{\prime}}$, and $u(t) \in \Re^{m}$ is the system input (i.e., control output); and $A_{i}(\cdot), B_{i}(\cdot)$ and $C(\cdot)$ are, respectively, $n \times n, n \times m$ and $n^{\prime} \times n$ matrices whose elements are known to be piecewise-continuous (PC) and real-valued functions defined on positive real space, $\Re_{+}$; in other words, they are matrix-valued functions on $\Re_{+}$of class PC. We then assume the desired controller is a rule-based fuzzy controller in the form of $R^{i}:$ If $y_{1}$ is $S_{1 i}, \cdots, y_{n^{\prime}}$ is $S_{n^{\prime} i}$

$$
\text { then } u(t)=r_{i}(t), \quad i=1, \cdots, \delta,
$$

where $y_{1}, \cdots, y_{n^{\prime}}$ are the elements of output vector $Y(t)$, $S_{1 i}, \cdots, S_{n^{\prime} i}$ are the input fuzzy terms in the $i$ th control rule, and the plant input (i.e., control output) vector $u(t)$ or $r_{i}(t)$ is in $\Re^{m}$ space. Then, the quadratic optimal fuzzy control problem is described as follows:

PROBLEM 1. Given the rule-based fuzzy system in (1) with $X \overline{\left(t_{0}\right)=X_{0}} \in \Re^{n}$ and a rule-based fuzzy controller in (2), find the individual optimal control law, $r_{i}^{*}(\cdot), i=1, \cdots, \delta$, such that the composed optimal controller $u^{*}(\cdot)$ can minimize the quadratic cost functional $J(u(\cdot))$ over all possible inputs $u(\cdot)$ of class PC

$$
\begin{aligned}
J(u(\cdot))= & \int_{t_{0}}^{t_{1}}\left[X^{T}(t) L(t) X(t)+u^{T}(t) u(t)\right] d t \\
& +X^{T}\left(t_{1}\right) Q X\left(t_{1}\right) \quad \text { (continuous) } \\
J(u(\cdot))= & \sum_{t=t_{0}}^{N-1}\left[X^{T}(t) L(t) X(t)+u^{T}(t) u(t)\right] \\
& +X^{T}(N) Q X(N) \quad \text { (discrete) }
\end{aligned}
$$

where $X^{T}(t) L(t) X(t), X^{T}\left(t_{1}\right) Q X\left(t_{1}\right)$ and $X^{T}(N) Q X(N)$ are state-trajectory penalties with both $L(t)$ and $Q$ belonging to symmetric positive semidefinite $n \times n$ matrices, and $u^{T}(t) u(t)$ is fuel consumption.

The grounding on distributed fuzzy subsystems and rule-based fuzzy controller forces the researchers to find the controller $u^{*}(\cdot)$, which can achieve global minimum effect under quadratic performance consideration defined on the entire fuzzy system and fuzzy controller. Thus this issue has not been attacked directly even though the T-S type fuzzy model has been available for many years. Wang [12] tried to break the deadlock by considering a linear system (instead of a fuzzy system) combined with a fuzzy controller. Tanaka and coworkers [21], [22] developed the LMI-based fuzzy control by assuming a local-linear-feedback-gain control structure. However, the quadratic optimal fuzzy control issue, in fact, remains fully open.

In the remainder of this section, the discrete-time case will be adopted for developing the local-concept-based optimization technology. From the essence of the dynamic programming formalism, the operation of minimizing $J(u(\cdot))$ in (4) can be decomposed as follows:

$$
\begin{aligned}
\min _{u_{\left[t_{0}, N-1\right]}} J(u(\cdot)) & \\
=\min _{u_{[t, N-1]}}\{ & \sum_{t}^{N-1}\left(X_{l}^{T} L_{l} X_{l}+u_{l}^{T} u_{l}\right)+X_{N}^{T} Q X_{N} \\
& \left.+\min _{u_{\left[t_{0}, t\right]}} \sum_{t_{0}}^{t}\left(X_{l}^{T} L_{l} X_{l}+u_{l}^{T} u_{l}\right)\right\}
\end{aligned}
$$

where we use the lower index to denote time dependence for notation simplification, i.e., $X_{l}$ for $X(l)$. Hence, The quadratic optimization problem is, in fact, a successively ongoing dynamic problem with regard to the state resulting from the previous decision, i.e., the initial state (at time step $t$ ) $X_{0_{t}}=X_{t}^{*}$. Moreover, according to the signal flow of a fuzzy inference system [23], we know, at any time step $l$, the overall behavior of the fuzzy system can be captured by fuzzily blending all the fuzzy subsystems; in other words, the entire T-S type fuzzy system in (1) can be represented as

$$
\begin{aligned}
X_{l+1}=\sum_{i=1}^{r} h_{i}\left(X_{l}\right)\left(A_{i_{l}} X_{l}+B_{i_{l}} u_{l}\right), & \\
l & \in[t, N-1], t \in\left[t_{0}, N-1\right]
\end{aligned}
$$

with

$$
u_{l}=\sum_{i=1}^{\delta} w_{i}\left(Y_{l}\right) r_{i_{l}}
$$

and $X_{0_{t}}=X_{t}^{*} \in \Re^{n}$, where $h_{i}\left(X_{l}\right)$ and $w_{i}\left(Y_{l}\right)$ denote, respectively, the normalized firing strength of the $i$ th rule of the fuzzy model and of the $i$ th fuzzy control rule; i.e., $h_{i}\left(X_{l}\right)=$ $\alpha_{i} / \sum_{i=1}^{r} \alpha_{i}$ with

$$
\alpha_{i}=\prod_{j=1}^{n} \mu_{T_{j i}}\left(X_{l}\right)
$$


where $\mu_{T_{j i}}\left(X_{l}\right)$ is the membership function of fuzzy term $T_{j i}$, and $w_{i}\left(Y_{l}\right)=\beta_{i} / \sum_{i=1}^{\gamma} \beta_{i}$ with

$$
\beta_{i}=\prod_{j=1}^{l} \mu_{S_{j i}}\left(Y_{l}\right)
$$

where $\mu_{S_{j i}}\left(Y_{l}\right)$ is the membership function of fuzzy term $S_{j i}$.

Therefore, the optimization dynamic issue is on successively finding the optimal global decision (optimal controller) $u_{t}^{*}$ for minimizing the cost functional

$$
\begin{aligned}
& J_{t}(u(\cdot))=\sum_{t}^{N-1}\left(X_{l}^{T} L_{l} X_{l}+u_{l}^{T} u_{l}\right)+X_{N}^{T} Q X_{N}, \\
& t \in\left[t_{0}, N-1\right]
\end{aligned}
$$

and estimating $X_{t+1}^{*}$ with regard to the initial state $X_{t}^{*}$; and then, with the new initial state $X_{t+1}^{*}$, resolving $u_{t+1}^{*}$ to minimize $J_{t+1}(u(\cdot))$. In other words, the quadratic optimal fuzzy control problem in Problem 1 can be restated as the following dynamic problem:

PROBLEM 2 . Given the fuzzy system in (6) with

$$
u_{l}=\sum_{i=1}^{\delta} w_{i}\left(Y_{l}\right) r_{i_{l}}
$$

successively find the optimal global decision, $u_{t}^{*}$, for minimizing the quadratic cost functional $J_{t}(u(\cdot))$ in (7), where the initial state is the optimal state resulting from the previous decision, i.e., $X_{0_{t_{0}}}=X_{0}$ and $X_{0_{t}}=X_{t}^{*}, t \in\left[t_{0}+1, N-1\right]$.

As we know, the energy of the entire fuzzy system is the summatin of the energy of each fuzzy subsystem. Hence, based on the additive property of energy, we know that, at any time step $t$, if we can find the optimal local decision (optimal control law) for minimizing $J_{t}(u(\cdot))$ in (7) with regard to the fuzzy subsystem

$$
X_{l+1}=A_{i_{l}} X_{l}+B_{i_{l}} u_{l}, \quad l \in[t, N-1], \quad i=1, \cdots, r
$$

then their composed global decision can be a global minimizer of the total cost, $J_{t}(u(\cdot))$, with regard to the fuzzy system in (6). For clarity, since $u_{t}^{*}$ is only a variable to be solved irrespective of the aforementioned local optimization problem or of the global optimization issue in Problem 2, we can use $r_{i_{t}}^{*}$ to denote the optimal local decision of the $i$ th fuzzy subsystem. Hence, based on the local viewpoint of the global optimal fuzzy control, we know that solving the quadratic optimal control problem in Problem 2 is to find only one corresponding optimal solution of the fuzzy controller for each rule of the fuzzy model. Thereupon, both the fuzzy model and admissible fuzzy controller have, more precisely, the same input variables and same input space partition, and there exists only one optimal fuzzy control rule for each fuzzy subsystem described by a fuzzy rule in the fuzzy model; that is

$$
\begin{gathered}
\text { (plant) } R^{i} \text { : If } x_{1} \text { is } T_{1 i}, \cdots, x_{n} \text { is } T_{n i}, \\
\text { then } S X(t)=A_{i}(t) X(t)+B_{i}(t) u(t), \\
Y(t)=C(t) X(t) \\
\text { (controller) } R^{i} \text { : If } x_{1} \text { is } T_{1 i}, \cdots, x_{n} \text { is } T_{n i}, \\
\text { then } u(t)=r_{i}(t), \quad i=1, \cdots, r
\end{gathered}
$$

and a fuzzy subsystem and fuzzy control rule have a one-to-one correspondence ( $i$ th-rule-to- $i$ th-rule). Therefore, the optimal global decisions $\left[u_{l}^{*}\right]_{t_{0}}^{N-1}$ in Problem 1 can be regarded as a series of optimal global decision $u_{t}^{*}$ based on the following successively ongoing local quadratic optimal issue with the initial state resulting from the previous decision.

PROBLEM $\underline{3 .}$ Given the fuzzy subsystem

$$
X_{l+1}=A_{i_{l}} X_{l}+B_{i_{l}} r_{i_{l}}, \quad l \in[t, N-1], \quad i=1, \cdots, r
$$

with the initial state resulting from the previous decision, i.e., $X_{0_{t}}=X_{t}^{*}$

1) find the optimal local decision at time-step $t, r_{i_{t}}^{*}$, for minimizing the cost functional

$$
J_{t}\left(r_{i}(\cdot)\right)=\sum_{t}^{N-1}\left(X_{l}^{T} L_{l} X_{l}+r_{i_{l}}^{T} r_{i_{l}}\right)+X_{N}^{T} Q X_{N} ;
$$

2) obtain the optimal global decision at time-step $t, u_{t}^{*}$, for minimizing the cost functional $J_{t}(u(\cdot))$ in (7) by fuzzily blending each local decision, i.e.,

$$
u_{t}^{*}=\sum_{i=1}^{r} h_{i}\left(X_{t}^{*}\right) r_{i_{t}}^{*} .
$$

Notice that the next-decision initial state is

$$
X_{t+1}^{*}=\sum_{i=1}^{r} h_{i}\left(X_{t}^{*}\right)\left(A_{i_{t}} X_{t}^{*}+B_{i_{t}} r_{i_{t}}^{*}\right)
$$

instead of $X_{t+1}^{*}$ in (6), since there exists the one-to-one relationship between each fuzzy subsystem and the corresponding fuzzy controller.

\section{OPTIMAL FuZZY CONTROLLER DESIGN}

We shall design the optimal controllers for the continuous-time systems in Section III-A and for discrete-time systems in Section III-B.

\section{A. Optimal Fuzzy Controller for Continuous-Time Fuzzy System}

Since the local fuzzy system (i.e., fuzzy subsystem) is linear, its quadratic optimization problem is the same as the general linear quadratic (LQ) issue [24]. Therefore, solving the optimal control problem for fuzzy subsystem can be achieved by simply generalizing the classical theorem in Proposition 4 in the Appendix from the deterministic case to fuzzy case. We summarize this generalization result here.

Theorem 1 (Solution of the Standard Fuzzy LQ Problem): For the fuzzy system in (1) and fuzzy controller in (2), let $A_{i}(t), B_{i}(t), C(t), L(t)=L^{T}(t) \geq 0, Q=Q^{T} \geq 0$, be given matrices. If there exists on $\left[t_{0}, t_{1}\right]$ an $n \times n$ symmetric positive semidefinite solution $\pi^{i}\left(t, Q, t_{1}\right)$ to the matrix Riccati differential equation

$$
\begin{aligned}
\dot{K}(t)= & -A_{i}^{T}(t) K(t)-K(t) A_{i}(t) \\
& +K(t) B_{i}(t) B_{i}^{T}(t) K(t)-L(t)
\end{aligned}
$$

where the final value of the dependent variable $K(t), K\left(t_{1}\right)$, is equal to the final state penalty index $Q$, and $t \in\left[t_{0}, t_{1}\right]$, then there exists a local optimal fuzzy control law

$$
r_{i}^{*}(t)=-B_{i}^{T} \pi^{i}\left(t, Q, t_{1}\right) X^{*}(t), \quad i=1, \cdots, r
$$


where $X^{*}(t)$ is the corresponding optimal state trajectory. And, the corresponding global minimizer is

$$
u^{*}(t)=\sum_{i=1}^{r} h_{i}\left(X^{*}(t)\right) r_{i}^{*}(t)
$$

which minimizes $J(u(\cdot))$ in (3). The resulting optimal closed-loop system dynamics is described by

$\dot{X}^{*}(t)=\sum_{i=1}^{r} h_{i}\left(X^{*}(t)\right)\left[A_{i}(t)-B_{i}(t) B_{i}^{T}(t) \pi^{i}\left(t, Q, t_{1}\right)\right] X^{*}(t)$,

$$
t \in\left[t_{0}, t_{1}\right]
$$

with $X\left(t_{0}\right)=X_{0}$.

Proof: This theorem obviously holds with Proposition 4 in the Appendix.

The above theorem considers that the horizon $t_{1}$ is fixed and $t_{0} \in\left[0, t_{1}\right)$ is arbitrary. Does the controller exist when the horizon goes to infinity? For the general LQ problem, the answer is positive if the system is time-invariant and well-behaved, i.e., completely controllable and completely observable. Now, we assume our fuzzy subsystem is linear time-invariant and well-behaved. In this case, the results below for each fuzzy subsystem are similar to those for a deterministic system described by Propositions 5 and 6 in the Appendix.

Theorem 2: For the fuzzy system in (1) and fuzzy controller in (2), let $A_{i}, B_{i}, C, L$ be given constant matrices and $L=$ $C^{T} C$. If $\left(A_{i}, B_{i}\right)$ is completely controllable (c.c.) and $\left(A_{i}, C\right)$ is completely observable (c.o.) for $i=1, \cdots, r$, then

1) there exists a unique $n \times n$ symmetric positive semidefinite solution, $\pi_{\infty}^{i}$, of the steady-state Riccati equation (S.S.R.E.)

$$
A_{i}^{T} K+K A_{i}-K B_{i} B_{i}^{T} K+C^{T} C=0
$$

2) the asymptotically local optimal fuzzy control law is

$$
r_{i}^{*}(t)=-B_{i}^{T} \pi_{\infty}^{i} X^{*}(t), \quad i=1, \cdots, r
$$

and their "blending" global minimizer $u^{*}(t)$ in (14) minimizes

$$
J(u(\cdot))=\int_{0}^{\infty}\left[X^{T}(t) L X(t)+u^{T}(t) u(t)\right] d t
$$

3) and the optimal local feedback fuzzy subsystem

$$
\dot{X}^{*}(t)=\left(A_{i}-B_{i} B_{i}^{T} \pi_{\infty}^{i}\right) X^{*}(t)
$$

is asymptotically and exponentially stable.

Proof: This theorem obviously holds with Propositions 5 and 6 in the Appendix.

\section{B. Optimal Fuzzy Controller for Discrete-Time Fuzzy System}

In the discrete-time system, the generalization of optimal control theory from general "deterministic" system to "fuzzy" subsystem is also practicable. The following theorem converts the result of the general LQ problem in the Appendix into its fuzzy optimal counterpart.

Theorem 3: For the fuzzy system in (1) and fuzzy controller in (2), let $A_{i}(t), B_{i}(t), C(t), L(t)=L^{T}(t) \geq 0, Q=Q^{T} \geq 0$ be given matrices. If there exists a symmetric positive semidefinite solution $\pi^{i}(t)$ to the following matrix Riccati difference equation:

$$
\begin{aligned}
V(t)= & -A_{i}^{T}(t) V(t+1) B_{i}(t)\left[I_{n}+B_{i}^{T}(t) V(t+1) B_{i}(t)\right]^{-1} \\
& \times B_{i}^{T}(t) V(t+1) A_{i}(t) \\
& +A_{i}^{T}(t) V(t+1) A_{i}(t)+L(t), \quad t=t_{0}, \cdots, N-1
\end{aligned}
$$

with $V(N)=0$ and $I_{n}$ the identity matrix of dimension $n$, then there exists a local optimal fuzzy control law

$$
\begin{aligned}
r_{i}^{*}(t)= & -\left[I_{n}+B_{i}^{T}(t) \pi^{i}(t+1) B_{i}(t)\right]^{-1} \\
& \times B_{i}^{T}(t) \pi^{i}(t+1) A_{i}(t) X^{*}(t), \quad i=1, \cdots, r
\end{aligned}
$$

and the resultant global controller is

$$
u^{*}(t)=\sum_{i=1}^{r} h_{i}\left(X^{*}(t)\right) r_{i}^{*}(t)
$$

which minimizes $J(u(\cdot))$ in (4). Moreover, the optimal trajectory is

$$
\begin{aligned}
& X^{*}(t+1) \\
& \quad=\sum_{i=1}^{r} h_{i}\left(X^{*}(t)\right)\left[I_{n}+B_{i}(t) B_{i}^{T}(t) \pi^{i}(t+1)\right]^{-1} A_{i}(t) X^{*}(t) .
\end{aligned}
$$

Notice that, using standard matrix manipulations, the matrix Riccati difference equation in (20) can be rewritten as

$$
\begin{aligned}
V(t)= & L(t)+A_{i}^{T}(t) V(t+1) \\
& \times\left[I_{n}+B_{i}(t) B_{i}^{T}(t) V(t+1)\right]^{-1} A_{i}(t) .
\end{aligned}
$$

Proof: This theorem obviously holds with Proposition 8 in the Appendix.

Similarly, if the fuzzy subsystem is linear time-invariant and well-behaved, meaning that it is stabilizable and detectable, we still can obtain infinite-horizon optimal controller as described in the following theorem.

Theorem 4: For the fuzzy system in (1) and fuzzy controller in (2), let $A_{i}, B_{i}, C, L$ be given constant matrices and $L=$ $C^{T} C$. If $\left(A_{i}, B_{i}\right)$ is stabilizable and $\left(A_{i}, C\right)$ is detectable for $i=1, \cdots, r$, then

1) there exists a unique symmetric positive semidefinite solution $\pi^{i}(\infty)$ of the following S.S.R.E.:

$$
\begin{aligned}
V(\infty)= & L+A_{i}^{T} V(\infty)\left[I_{n}+B_{i} B_{i}^{T} V(\infty)\right]^{-1} A_{i} \\
V(\infty)= & L+A_{i}^{T} V(\infty) A_{i}-A_{i}^{T} V(\infty) B_{i} \\
& \times\left[I_{n}+B_{i}^{T} V(\infty) B_{i}\right]^{-1} B_{i}^{T} V(\infty) A_{i}
\end{aligned}
$$

2 ) the asymptotically local optimal fuzzy control law is

$$
\begin{array}{r}
r_{i}^{*}(t)=-\left[I_{n}+B_{i}^{T} \pi^{i}(\infty) B_{i}\right]^{-1} B_{i}^{T} \pi^{i}(\infty) A_{i} X^{*}(t), \\
t=t_{0}, \cdots, N-1
\end{array}
$$

and the resultant global controller $u^{*}(t)$ in (22) minimizes

$$
J(u(\cdot))=\sum_{t=t_{0}}^{\infty}\left[X^{T}(t) L X(t)+u^{T}(t) u(t)\right]
$$


3) moreover, the optimal local feedback fuzzy subsystem

$$
X^{*}(t+1)=\left[I_{n}+B_{i} B_{i}^{T} \pi^{i}(\infty)\right]^{-1} A_{i} X^{*}(t)
$$

is asymptotically and exponentially stable.

Proof: This theorem obviously holds with Proposition 9 in the Appendix.

\section{STABILITY AND GAin MARgin}

In this section, we are concerned with the stability of the global closed-loop system with the optimal fuzzy controller designed in the preceding section. We shall show that the controller resulting from the infinite-horizon optimal control problem gives not merely an asymptotically stable closed-loop system, but one with a prescribed degree of stability. Furthermore, we also define the term gain margin to discuss what range of the feedback gain we can enlarge under the stability consideration.

\section{A. Global Stability}

The entire feedback fuzzy system is nonlinear, even though the subsystem is linear. We can thus apply the so-called converse theorem of Lyapunov stability theory in the nonlinear system [25] to our fuzzy system. This theorem is given in the following proposition.

Proposition 1: Consider the system

$$
\dot{X}(t)=F(X(t))
$$

where $F$ is a $\mathcal{C}^{2}$ function, i.e., an ordered 2-tuple complexvalued function, and $F\left(X_{o}\right)=0$. Define

$$
G=\left.(\partial F / \partial X)\right|_{X_{o}}
$$

then $X_{o}$ is an exponentially stable equilibrium of the system if and only if the linearized system

$$
\dot{Z}(t)=G Z(t)
$$

is (globally) exponentially stable. Additionally, if $G$ is defined as above and if all the eigenvalues of $G$ have negative real parts, then $X_{0}$ is an exponentially stable equilibrium.

Via the converse theorem, the stability analysis of a nonlinear system is coincidental with that of the linearized system. For the T-S type fuzzy system, a locally linearized system from the global system in (6), we know that the linearized matrix $G$ in the above proposition at some point $X_{o}$ is $\sum_{i=1}^{r} h_{i}\left(X_{o}\right) A_{i}(t)$. Hence, the term $\sum_{i=1}^{r} h_{i}(X(t)) A_{i}(t)$ fully handles the stability of the fuzzy system.

From Theorems 2 and 4, we know that in the infinite-horizon optimal control problem, if the local fuzzy system is time-invariant and well-behaved, the local feedback fuzzy system is asymptotically and exponentially stable no matter whether the system is continuous or discrete. Now our strategy is to ground on this nice local feature and step for the global system by the spectral mapping theorem in [26], which says that the spectrum of a analytic function of an operator is the analytic function of the spectrum of the operator. This will be exposed on the following theorem.
Theorem 5: For the time-invariant fuzzy system in (1) and fuzzy controller in (2), if $\left(A_{i}, B_{i}\right)$ is c.c. and $\left(A_{i}, C\right)$ is c.o. for $i=1, \cdots, r$, then

1) the optimal feedback fuzzy system

$$
\dot{X}^{*}(t)=\sum_{i=1}^{r} h_{i}\left(X^{*}(t)\right)\left[A_{i}-B_{i} B_{i}^{T} \pi_{\infty}^{i}\right] X^{*}(t)
$$

is exponentially stable;

2) the total energy of system output is finite

$$
\int_{0}^{\infty}\|Y(t)\|^{2} d t<\infty
$$

Proof:

1) Via the converse theorem, we know the stability of the resultant feedback fuzzy system in (30) concurs with that of the linearized fuzzy system (with respect to $X_{o}$ )

$$
\dot{X}^{*}(t)=\sum_{i=1}^{r} h_{i}\left(X_{o}\right)\left[A_{i}-B_{i} B_{i}^{T} \pi_{\infty}^{i}\right] X^{*}(t) .
$$

For clarity, we introduce the notation $A_{c i}$ to denote the local feedback system matrix. Then, we know, via Theorem 2, that each feedback fuzzy subsystem is exponentially stable, which means the spectrum of $A_{c i}, i=1, \cdots, r$, denoted by $\sigma\left[A_{c i}\right]$, is located in the open left-half plane of the complex space $\mathcal{C}_{-}^{o}$, i.e., $\sigma\left[A_{c i}\right] \subset \mathcal{C}_{-}^{o}, i=1, \cdots, r$. Accordingly, we have

$$
\sigma\left[h_{i}\left(X_{o}\right) A_{c i}\right] \subset \mathcal{C}_{-}^{\circ}, \quad i=1, \cdots, r
$$

via the spectral mapping theorem and $h_{i}\left(X_{o}\right) \in[0,1]$ for all $X_{\circ} \in \Re^{n}$. Hence, the zero solution of $\dot{X}(t)=$ $h_{i}\left(X_{o}\right) A_{c i} X(t)$ on $t \geq t_{0}$ is exponentially stable; in other words, there exists constants $a_{i}>0$ and $m_{i}>0$ such that for all $t_{0} \in \Re_{+}$

$$
\begin{aligned}
&\left\|e^{h_{i}\left(X_{o}\right) A_{c i}\left(t-t_{0}\right)}\right\| \leq m_{i} e^{-a_{i}\left(t-t_{0}\right)}, \\
& \forall t \geq t_{0}, \quad i=1, \cdots, r .
\end{aligned}
$$

Then, the state transition matrix $\phi\left(t, t_{0}\right)$ of the linearized fuzzy system in (31) is

$$
\begin{aligned}
\left\|\phi\left(t, t_{0}\right)\right\| & =\left\|e^{\sum_{i=1}^{r} h_{i}\left(X_{o}\right) A_{c i}\left(t-t_{0}\right)}\right\| \\
& \leq \prod_{i=1}^{r}\left\|e^{h_{i}\left(X_{o}\right) A_{c i}\left(t-t_{0}\right)}\right\| \\
& \leq \prod_{i=1}^{r} m_{i} e^{-a_{i}\left(t-t_{0}\right)} \leq m e^{-a\left(t-t_{0}\right)}
\end{aligned}
$$

where $m \triangleq \prod_{i=1}^{r} m_{i}>0$ and $a \triangleq \sum_{i=1}^{r} a_{i}>0$. Therefore, the linearized fuzzy system in (31) and also the feedback fuzzy system in (30) are exponentially stable. Hence, we can conclude that the entire continuous fuzzy system is exponentially stable if each continuous fuzzy subsystem is exponentially stable.

2) From the above proof, we know the entire feedback fuzzy system in (30) is exponential stable and, hence, also ensure to be uniformly asymptotically stable. Therefore, for 
all $X\left(t_{0}\right)=X_{0} \in \Re^{n}$ and $t_{0} \in \Re_{+}$, the state $X(t)$ satisfies a) and b) in the following.

a) The range of mapping from $t$ to $X(t)$ is bounded on $t>0$ uniformly, i.e.,

$$
\exists k<\infty \text { s.t. }\|X(t)\|<k, \quad \forall t>0 .
$$

b) The range of mapping from $t$ to $X(t)$ tends to zero as $t \rightarrow \infty$ uniformly, i.e.,

$\forall \epsilon>0, \quad \exists T(\epsilon)>0$ s.t. $\|X(t)\| \leq \epsilon, \quad \forall t>T(\epsilon)$.

Therefore, with $\sum_{i=1}^{r} h_{i}(X(t))=1$,

$$
\begin{aligned}
\int_{0}^{\infty}\|Y(t)\|^{2} d t & =\int_{0}^{\infty}\left\|\sum_{i=1}^{r} h_{i}(X(t)) C X(t)\right\|^{2} d t \\
= & \int_{0}^{\infty}\|C X(t)\|^{2} d t \\
\leq & \int_{0}^{\infty}\|C\|^{2}\|X(t)\|^{2} d t \\
= & \int_{0}^{T}\|C\|^{2}\|X(t)\|^{2} d t \\
& +\int_{T}^{\infty}\|C\|^{2}\|X(t)\|^{2} d t .
\end{aligned}
$$

From a) and b), we know that these two integrates are both finite, and accordingly we have

$$
\int_{0}^{\infty}\|Y(t)\|^{2} d t<\infty
$$

However, we cannot yet demonstrate that there exists such close stability relationship between the entire closed-loop system and the local feedback system for the discrete-time case. Therefore, we can only use Lyapunov's direct method or linear matrix inequality method [8] to analyze the stability of the overall feedback discrete-time fuzzy system.

\section{B. Stabilization to Any Desired Degree}

So far, we have examined the stability of the closed-loop system. We now attempt to show that the constructed optimal fuzzy controller can stabilize the entire fuzzy system to any desired degree. That is, for some prescribed constant $\alpha>0$, the state $X(t)$ approaches zero at least by the rate of $e^{-\alpha t}$. Of course, the larger the desired degree of stability, the more stable the closed-loop system. However, a high degree of closed-loop stability may only be achieved at excessive control energy consumption. Before showing this, we need the following two lemmas.

Lemma 1: For a system $R$ described by

$$
\begin{aligned}
\dot{X}(t) & =A X(t)+B u(t) \\
Y(t) & =C X(t)
\end{aligned}
$$

where $A, B$, and $C$ are $n \times n, n \times m$, and $n^{\prime} \times n$ matrices (i.e., $R=[A, B, C]) .(A, B)$ c.c. is equivalent to $\left(\alpha I_{n}+A, B\right)$ c.c. for any complex value $\alpha$, i.e.,

$$
(A, B) \text { c.c. } \Leftrightarrow\left(\alpha I_{n}+A, B\right) \text { c.c., } \quad \forall \alpha \in \mathcal{C} .
$$

Proof:

1) $(A, B)$ is c.c. if and only if $\operatorname{rank}\left[B A B \cdots A^{n-1} B\right]=n$. Let $\hat{B} \triangleq e^{-\alpha t} B$, then

$$
\begin{aligned}
& \operatorname{rank}\left[\hat{B} \quad A \hat{B} \cdots A^{n-1} \hat{B}\right] \\
& =\operatorname{rank}\left\{e^{-\alpha t}\left[\begin{array}{llll}
B & A B & \cdots & A^{n-1} B
\end{array}\right]\right\}
\end{aligned}
$$



which means $(A, B)$ is c.c. if and only if $(A, \hat{B})$ is c.c.

2) Now, consider two systems

$$
\begin{aligned}
\dot{X} & =A X+\hat{B} u \\
\dot{\hat{X}} & =\left(\alpha I_{n}+A\right) \hat{X}+B u .
\end{aligned}
$$

If we let $\hat{X}=e^{\alpha t} X$, via basic differential operation, it is evident that (32) and (33) are algebraically equivalent for any $\alpha \in \mathcal{C}$; i.e., they are related by a nonsingular linear transformation $e^{\alpha t}$. Therefore, (32) c.c. is equivalent to (33) c.c., i.e.,

$$
(A, \hat{B}) \text { c.c. } \Leftrightarrow\left(\alpha I_{n}+A, B\right) \text { c.c., } \quad \forall \alpha \in \mathcal{C} .
$$

From 1) and 2), we conclude that Lemma 1 holds.

Lemma 2: For a system $R=[A, B, C],(A, C)$ c.o. is equivalent to $\left(\alpha I_{n}+A, C\right)$ c.o. for any complex value $\alpha$, i.e.,

$$
(A, C) \text { c.o. } \Leftrightarrow\left(\alpha I_{n}+A, C\right) \text { c.o. }, \quad \forall \alpha \in \mathcal{C} .
$$

Proof: The proof is similar to the proof of Lemma 1.

Now, we deduce Theorem 6 using the above two lemmas.

Theorem 6: For the fuzzy system in (1) and fuzzy controller in (2), let $A_{i}, B_{i}, C, L$ be given constant matrices and $L=C^{T} C$ in (18). If $\left(A_{i}, B_{i}\right)$ is c.c. and $\left(A_{i}, C\right)$ is c.o. for $i=1, \cdots, r$, then the fuzzy system can be stabilized to any desired degree of stability; in other words, the state of the modified feedback fuzzy system

$$
\dot{X}^{*}(t)=\sum_{i=1}^{r} h_{i}\left(X^{*}(t)\right)\left(A_{i}-B_{i} B_{i}^{T} \hat{\pi}_{\infty}^{i}(\alpha)\right)
$$

approaches 0 at least by the rate of $e^{-\alpha t}$, where $\alpha$ is any positive real number, $h_{i}\left(X^{*}(t)\right)$ is the normalized firing strength (i.e., $\left.\sum_{i=1}^{r} h_{i}\left(X^{*}(t)\right)=1\right)$, and $\hat{\pi}_{\infty}^{i}(\alpha)$ is the positive-semidefinite solution of the modified S.S.R.E.

$\left(\alpha I_{n}+A_{i}\right)^{T} K(\alpha)+K(\alpha)\left(\alpha I_{n}+A_{i}\right)-K(\alpha) B_{i} B_{i}^{T} K(\alpha)+L$

where $K(\alpha)$ is the dependent variable of the algebraic equation.

Proof:

1) Via the converse theorem, we know the stability of the modified feedback fuzzy system in (34) concurs with that of the linearized fuzzy system (with respect to $X_{o}$ )

$$
\dot{X}^{*}(t)=\sum_{i=1}^{r} h_{i}\left(X_{o}\right)\left(A_{i}-B_{i} B_{i}^{T} \hat{\pi}_{\infty}^{i}(\alpha)\right) X^{*}(t) .
$$

Hence, we shall show that all the eigenvalues of the linearized fuzzy system in the above have real part smaller than $-\alpha$, i.e.,

$$
\Re_{e}\left\{\lambda\left[\sum_{i=1}^{r} h_{i}\left(X_{o}\right)\left(A_{i}-B_{i} B_{i}^{T} \hat{\pi}_{\infty}^{i}(\alpha)\right)\right]\right\}<-\alpha .
$$


We now consider the local quadratic optimal problem

$$
\begin{aligned}
\min \int_{t_{0}}^{\infty}\left[X^{t}(t) L X(t)+r_{i}^{t}(t) r_{i}(t)\right] \\
\text { w.r.t. }\left\{\begin{array}{l}
\dot{X}(t)=A_{i} X(t)+B_{i} r_{i}(t) \\
Y(t)=C X(t) .
\end{array}\right.
\end{aligned}
$$

Let $\hat{X}(t)=e^{\alpha t} X(t), \hat{Y}(t)=e^{\alpha t} Y(t)$, and $\hat{r}_{i}(t)=e^{\alpha t} r_{i}(t)$. Equation (37) can be rewritten as

$$
\begin{aligned}
& \min \int_{t_{0}}^{\infty} e^{-2 \alpha t}\left[\hat{X}^{t}(t) L \hat{X}(t)+\hat{r}_{i}^{t}(t) \hat{r}_{i}(t)\right] \\
& \text { w.r.t. }\left\{\begin{array}{l}
\dot{\hat{X}}(t)=\left(\alpha I_{n}+A_{i}\right) \hat{X}(t)+B_{i} \hat{r}_{i}(t) \\
\hat{Y}(t)=C \hat{X}(t) .
\end{array}\right.
\end{aligned}
$$

From Lemmas 1 and 2, we know that $\left(A_{i}, B\right)$ c.c. and $\left(A_{i}, C\right)$ c.o., $\forall i=1, \cdots, r$, if and only if $\left(\alpha I_{n}+A_{i}, B_{i}\right)$ c.c. and $\left(\alpha I_{n}+A_{i}, C\right)$ c.o., $\forall i=1, \cdots, r$. Hence, based on the linear quadratic theory, we know the local optimal feedback system for the modified fuzzy system in (38),

$$
\dot{X}^{*}(t)=\left(\alpha I_{n}+A_{i}-B_{i} B_{i}^{T} \hat{\pi}_{\infty}^{i}(\alpha)\right) X^{*}(t)
$$

is exponentially stable for all $i=1, \cdots, r$. Accordingly, from part 1) of the proof in Theorem 5, we know that the fuzzily blended global feedback fuzzy system

$$
\dot{X}^{*}(t)=\sum_{i=1}^{r} h_{i}\left(X^{*}(t)\right)\left(\alpha I_{n}+A_{i}-B_{i} B_{i}^{T} \hat{\pi}_{\infty}^{i}(\alpha)\right) X^{*}(t)
$$

and also the corresponding linearized global fuzzy system (with respect to $X_{o}$ ) are exponentially stable, where $\sum_{i=1}^{r} h_{i}\left(X^{*}(t)\right)=1$ for all $X^{*}(t) \in \Re^{n}$. Thereupon, we have

$$
\begin{aligned}
& \Re_{e}\left\{\lambda\left[\sum_{i=1}^{r} h_{i}\left(X_{o}\right)\left(\alpha I_{n}+A_{i}-B_{i} B_{i}^{T} \hat{\pi}_{\infty}^{i}(\alpha)\right)\right]\right\}<0 \\
& \Re_{e}\left\{\lambda\left[\alpha I_{n}+\sum_{i=1}^{r} h_{i}\left(X_{o}\right)\left(A_{i}-B_{i} B_{i}^{T} \hat{\pi}_{\infty}^{i}(\alpha)\right)\right]\right\}<0 \\
& \Re_{e}\left\{\lambda\left[\sum_{i=1}^{r} h_{i}\left(X_{o}\right)\left(A_{i}-B_{i} B_{i}^{T} \hat{\pi}_{\infty}^{i}(\alpha)\right)\right]\right\}<-\alpha
\end{aligned}
$$

with the aid of the spectral mapping theorem. This completes the proof.

\section{Gain Margin}

Furthermore, we shall examine another interesting property, gain margin, of the resultant closed-loop fuzzy system. Recall that the gain margin of a closed-loop system is the amount by which the loop gain can be changed until the system becomes unstable. If the loop gain can be increased without bound; that is, instability is not encountered no matter how large the loop gain becomes, then the closed-loop system is said to possess an infinite gain margin [24]. Thus far, for time-invariant wellbehaved continuous fuzzy subsystem, we know the designed global optimal controller, by (17) and (14), is

$$
u^{*}(t)=-\sum_{i=1}^{r} h_{i}\left(X^{*}(t)\right) B_{i}^{T} \pi_{\infty}^{i} X^{*}(t) .
$$

In order to measure the gain margin, we think of the following control law:

$$
u(t)=\left\{\beta\left[-\sum_{i=1}^{r} h_{i}(X(t)) B_{i}^{T} \pi_{\infty}^{i}\right]\right\} X(t), \quad \beta \geq 1 .
$$

Then, the gain margin of the closed-loop fuzzy system is defined as the amount by which $\beta$ can be increased until the system becomes unstable. In this case, the corresponding local control law $r_{i}(t)$ is

$$
r_{i}(t)=-\beta B_{i}^{T} \pi_{\infty}^{i} X(t) .
$$

Notice that in the case of $\beta>1$, this control law is no longer an optimal control law, i.e., $r_{i}(t) \neq r_{i}^{*}(t)$. Now, let

$$
\gamma_{i}(t) \triangleq r_{i}(t) / \beta=-B_{i}^{T} \pi_{\infty}^{i} X(t)
$$

and then we have

$$
\begin{aligned}
J\left(r_{i}(\cdot)\right) & =\int_{0}^{\infty}\left[X^{T}(t) L X(t)+r_{i}^{T}(t) r_{i}(t)\right] d t \\
& =\int_{0}^{\infty}\left[X^{T}(t) L X(t)+\beta^{2} \gamma_{i}^{T}(t) \gamma_{i}(t)\right] d t .
\end{aligned}
$$

We further consider

$$
\begin{array}{r}
J\left(\gamma_{i}(\cdot)\right)=\int_{0}^{\infty}\left[q X^{T}(t) L X(t)+\gamma_{i}^{T}(t) \gamma_{i}(t)\right] d t, \\
L=C^{T} C, q>0 .
\end{array}
$$

Notice that $J\left(r_{i}(\cdot)\right)=\beta^{2} \cdot J\left(\gamma_{i}(\cdot)\right)$ and $q=1 / \beta^{2}$. Comparing (42) to (41), we find that the larger the $\beta$, the smaller the $q$, which means that when $q$ goes to zero, the gain margin of the closed-loop fuzzy system becomes infinite.

We can include $q$ into the state penalty matrix $L$. From Theorem 2, for any $q>0$, the optimal control law with respect to (42) is

$$
\gamma_{i}^{*}(t)=-B_{i}^{T} \hat{\pi}_{\infty}^{i}(q) X^{*}(t)
$$

where $\hat{\pi}_{\infty}^{i}(q)$ satisfies the modified S.S.R.E.

$$
A_{i}^{T} K(q)+K(q) A_{i}+q L-K(q) B_{i} B_{i}^{T} K(q)=0
$$

where $K(q)$ is the dependent variable of the algebraic equation. We now first cite two important results in control theory [28] and apply them to the fuzzy feedback subsystem. We shall then find the gain margin of the entire closed-loop fuzzy system. The following propositions are cited from [28].

Proposition 2: Consider the infinite-horizon optimal control problem as follows.

Given a linear time-invariant system

$$
\dot{X}(t)=A X(t)+B u(t)
$$

find an optimal controller $u^{*}(t)$ to minimize

$$
J(u(\cdot))=\int_{0}^{\infty}\left[X^{T}(t) L X(t)+u^{T}(t) u(t)\right] d t
$$

with $Y(t)$ denoting the system output, where $Y(t)=C X(t)$ and $L=C^{T} C$. Now, if $(A, B)$ is c.c. and $(A, C)$ is c.o., then

1) the positive-semidefinite solution, denoted by $\hat{\pi}_{\infty}(q)$, of the modified S.S.R.E.

$$
A^{T} K(q)+K(q) A+q L-K(q) B B^{T} K(q)=0
$$

uniquely exists;

2) when $q$ goes to zero, i.e., when the closed-loop system possesses an infinite gain margin, the limit value of $\hat{\pi}_{\infty}(q)$ 
exists. Let $\hat{\pi}_{\infty}(0)$ denote this limit value. Furthermore, $\hat{\pi}_{\infty}(0)$ is the unique positive-semidefinite solution of the modified S.S.R.E.

$$
A^{T} K+K A-K B B^{T} K=0 .
$$

Proposition 3: Consider the same infinite-horizon optimal control problem as in Proposition 2. Assume $(A, B)$ is c.c. and $(A, C)$ is c.o., then

1) we can find an optimal control law

$$
u^{*}(t)=-B^{T} \pi_{\infty} X^{*}(t)
$$

where $\pi_{\infty}$ is the positive-semidefinite solution of the S.S.R.E.

$$
A^{T} K+K A-K B B^{T} K+L=0
$$

and the closed-loop fuzzy system possesses an infinite gain margin; i.e., the modified feedback system $[A-$ $\left.\beta B B^{T} \hat{\pi}_{\infty}(q)\right]$ is always stable for any $\beta \geq 1$, where $q=1 / \beta^{2}$ and $\hat{\pi}_{\infty}(q)$ is the positive-semidefinite solution of the modified S.S.R.E., (44).

2) Moreover, for any fixed $\beta$, the enlarged controller

$$
\hat{u}^{*}(t)=-\beta B^{T} \hat{\pi}_{\infty}(q) X^{*}(t)
$$

can still stabilize the modified system to any desired degree of stability; i.e., all the eigenvalues of $A-\beta B B^{T} \hat{\pi}_{\infty}(\alpha, q)$ have real parts smaller than $-\alpha$, where $\alpha$ could be any positive real number and $\hat{\pi}_{\infty}(\alpha, q)$ is the positive-semidefinite solution of the modified S.S.R.E.

$$
\begin{aligned}
\left(\alpha I_{n}+A\right)^{T} K(\alpha, q) & +K(\alpha, q)\left(\alpha I_{n}+A\right) \\
& +q L-K(\alpha, q) B B^{T} K(\alpha, q)=0
\end{aligned}
$$

where $K(\alpha, q)$ is the dependent variable of the algebraic equation.

Grounding on these propositions, we elicit the following fascinating fact.

Theorem 7: For the time-invariant fuzzy system in (1) and fuzzy controller in (2) with $L=C^{T} C$ in (18), if $\left(A_{i}, B_{i}\right)$ is c.c. and $\left(A_{i}, C\right)$ is c.o. for $i=1, \cdots, r$, then

1) we can find a fuzzy control law

$$
u^{*}(t)=-\sum_{i=1}^{r} h_{i}\left(X^{*}(t)\right) B_{i}^{T} \pi_{\infty}^{i} X^{*}(t)
$$

where $\pi_{\infty}^{i}$ is the positive-semidefinite solution of the S.S.R.E. in (16), and the resultant closed-loop fuzzy system possesses an infinite gain margin; i.e., the modified closed-loop fuzzy system

$\dot{X}(t)=\sum_{i=1}^{r} h_{i}(X(t))\left[A_{i}-\beta B_{i} B_{i}^{T} \hat{\pi}_{\infty}^{i}(q)\right] X(t)$

is always stable for any $\beta \geq 1$, where $q=1 / \beta^{2}$ and $\hat{\pi}_{\infty}^{i}(q)$ is the positive semidefinite solution of the modified S.S.R.E. in (43).

2) Moreover, for any fixed $\beta$, the enlarged controller

$$
\hat{u}^{*}(t)=-\sum_{i=1}^{r} h_{i}\left(X^{*}(t)\right) \beta B_{i}^{T} \hat{\pi}_{\infty}^{i}(q) X^{*}(t)
$$

can still stabilize the modified system to any desired degree of stability; in other words, the state of the modified feedback fuzzy system

$$
\dot{X}(t)=\sum_{i=1}^{r} h_{i}\left(X^{*}(t)\right)\left[A_{i}-\beta B_{i} B_{i}^{T} \hat{\pi}_{\infty}^{i}(\alpha, q)\right] X(t)
$$

approaches 0 at least by the rate of $e^{-\alpha t}$, where $\alpha$ could be any positive real number and $\hat{\pi}_{\infty}^{i}(\alpha, q)$ is the positivesemidefinite solution of the modified S.S.R.E.

$$
\begin{aligned}
& \left(\alpha I_{n}+A_{i}\right)^{T} K(\alpha, q)+K(\alpha, q)\left(\alpha I_{n}+A_{i}\right) \\
& \quad+q L-K(\alpha, q) B_{i} B_{i}^{T} K(\alpha, q)=0 .
\end{aligned}
$$

Proof: For clarity, we introduce the notations $A_{c i}(\beta)$ and $A_{c i}(\alpha, \beta)$ to denote, respectively, the local feedback system matrices in (45) and (46), i.e.,

$$
A_{c i}(\beta)=A_{i}-\beta B_{i} B_{i}^{T} \hat{\pi}_{\infty}^{i}(q)
$$

and

$$
A_{c i}(\alpha, \beta)=A_{i}-\beta B_{i} B_{i}^{T} \hat{\pi}_{\infty}^{i}(\alpha, q) .
$$

1) Since $\left(A_{i}, B_{i}\right)$ is c.c. and $\left(A_{i}, C\right)$ is c.o. for $i=1, \cdots, r$, we know, from 1) in Proposition 3 , that the modified closed-loop fuzzy subsystem is stable for any $\beta \geq 1$, i.e., $\sigma\left(A_{c i}(\beta)\right) \subset \mathcal{C}_{-}^{o}$ for all $i=1, \cdots, r$. Accordingly, by part 1) of the proof in Theorem 5, we know their fuzzily blended global system in (45) is exponentially stable.

2) Then fixing at any gain margin $\beta$, we shall show the state of the modified feedback fuzzy system in (46) and also that of the corresponding linearized fuzzy system (with respect to $X_{o}$ ) approaches 0 at least by the rate of $e^{-\alpha t}$ for all $\alpha>0$. In other words, we shall demonstrate

$$
\Re_{e}\left\{\lambda\left[\sum_{i=1}^{r} h_{i}\left(X_{o}\right)\left(A_{c i}(\alpha, \beta)\right)\right]\right\}<-\alpha .
$$

From 2) in Proposition 3, we have

$$
\Re_{e}\left\{\lambda\left[A_{c i}(\alpha, \beta)\right]\right\}<-\alpha, \quad \forall i=1, \cdots, r
$$

due to $\left(A_{i}, B_{i}\right)$ being c.c. and $\left(A_{i}, C\right)$ being c.o. Accordingly, via the spectral mapping theorem, we have

$\Re_{e}\left\{\lambda\left[\alpha I_{n}+A_{c i}(\alpha, \beta)\right]\right\}<0, \quad$ for $i=1, \cdots, r$

which results in that all local modified feedback fuzzy system

$\dot{X}(t)=\left(\alpha I_{n}+A_{c i}(\alpha, \beta)\right) X(t), \quad i=1, \cdots, r$

are exponentially stable. Moreover, we know, via part 1) of the proof in Theorem 5, that the fuzzily blended feedback fuzzy system

$$
\dot{X}(t)=\sum_{i=1}^{r} h_{i}(X(t))\left(\alpha I_{n}+A_{c i}(\alpha, \beta)\right) X(t)
$$

is exponentially stable. Furthermore, via the converse theorem, we have

$$
\Re_{e}\left\{\lambda\left[\sum_{i=1}^{r} h_{i}\left(X_{o}\right)\left(\alpha I_{n}+A_{c i}(\alpha, \beta)\right]\right\}<0\right.
$$


for all $X_{O} \in \Re^{n}$. Then, we obtain

$$
\Re_{e}\left\{\lambda\left[\sum_{i=1}^{r} h_{i}\left(X_{o}\right)\left(A_{c i}(\alpha, \beta)\right)\right]\right\}<-\alpha
$$

for all $\alpha>0$ and $X_{o} \in \Re^{n}$. Therefore, we know that the linearized feedback fuzzy system (with respect to $X_{\circ}$ )

$$
\dot{X}(t)=\sum_{i=1}^{r} h_{i}\left(X_{o}\right) X(t) A_{c i}(\alpha, \beta)
$$

has any degree of stability. Hence, the modified fuzzy system in (46) has any degree of stability as well.

\section{NUMERICAL SimulationS}

We consider a simple nonlinear mass-spring-damper mechanical system for continuous-time case, and an optimal backing up control of a computer simulated trunk-trailer for discrete-time case in order to illustrate the proposed optimal fuzzy control scheme and its theoretic aspect.

\section{A. Continuous-Time System}

A mass-spring-damper system can be formulated as

$$
M \ddot{x}+g(x, \dot{x})+f(x)=\phi(\dot{x}) u
$$

where $M$ is the mass and $u$ is the force; $f(x)$ and $g(x, \dot{x})$ are the nonlinear or uncertain terms with respect to the spring and the damper, respectively, and $\phi(\dot{x})$ is the nonlinear term with respect to the input term. We make the same assumptions as Tanaka et al. did in [3], and reformulate the system as

$$
\ddot{x}=-0.1 \dot{x}^{3}-0.02 x-0.67 x^{3}+u
$$

where $x \in\left[\begin{array}{ll}-1.5 & 1.5\end{array}\right]$ and $\dot{x} \in\left[\begin{array}{ll}-1.5 & 1.5\end{array}\right]$.

According to the study in [3], we describe this nonlinear system by the following T-S type fuzzy model:

$$
\begin{aligned}
& R^{1}: \text { If } x(t) \text { is } F_{1}^{1} \text { and } \dot{x}(t) \text { is } F_{2}^{1}, \\
& \text { then } \dot{X}(t)=A_{1} X(t)+B_{1} u(t) \\
& R^{2}: \text { If } x(t) \text { is } F_{1}^{1} \text { and } \dot{x}(t) \text { is } F_{2}^{2}, \\
& \text { then } \dot{X}(t)=A_{2} X(t)+B_{2} u(t) \\
& R^{3}: \text { If } x(t) \text { is } F_{1}^{2} \text { and } \dot{x}(t) \text { is } F_{2}^{1}, \\
& \text { then } \dot{X}(t)=A_{3} X(t)+B_{3} u(t) \\
& R^{4}: \text { If } x(t) \text { is } F_{1}^{2} \text { and } \dot{x}(t) \text { is } F_{2}^{2}, \\
& \text { then } \dot{X}(t)=A_{4} X(t)+B_{4} u(t)
\end{aligned}
$$

and the system output is $Y(t)=C X(t)$ with $C=I_{2}$ for every rule, where

$$
\begin{aligned}
& X(t)=[\dot{x}(t) x(t)]^{T}, \\
& A_{1}=\left[\begin{array}{cc}
0 & -0.02 \\
1 & 0
\end{array}\right], \quad A_{2}=\left[\begin{array}{cc}
-0.225 & -0.02 \\
1 & 0
\end{array}\right], \\
& A_{3}=\left[\begin{array}{cc}
0 & -1.5275 \\
1 & 0
\end{array}\right], \quad A_{4}=\left[\begin{array}{cc}
-0.225 & -1.5275 \\
1 & 0
\end{array}\right], \\
& B_{i}=\left[\begin{array}{ll}
1 & 0
\end{array}\right]^{T}, \quad i=1, \cdots, 4
\end{aligned}
$$

and the membership functions of the precondition parts of the fuzzy rules are

$$
\begin{aligned}
& F_{1}^{1}(x(t))=1-\frac{x^{2}(t)}{2.25} \\
& F_{1}^{2}(x(t))=1-F_{1}^{1}(x(t))=\frac{x^{2}(t)}{2.25} \\
& F_{2}^{1}(\dot{x}(t))=1-\frac{\dot{x}^{2}(t)}{2.25} \\
& F_{2}^{2}(\dot{x}(t))=1-F_{2}^{1}(x(t))=\frac{\dot{x}^{2}(t)}{2.25}
\end{aligned}
$$

We further assume our fuzzy controller is

$$
\begin{aligned}
& R^{1}: \text { If } x(t) \text { is } F_{1}^{1} \text { and } \dot{x}(t) \text { is } F_{2}^{1}, \text { then } u(t)=r_{1}(t) \\
& R^{2}: \text { If } x(t) \text { is } F_{1}^{1} \text { and } \dot{x}(t) \text { is } F_{2}^{2}, \text { then } u(t)=r_{2}(t) \\
& R^{3}: \text { If } x(t) \text { is } F_{1}^{2} \text { and } \dot{x}(t) \text { is } F_{2}^{1}, \text { then } u(t)=r_{3}(t) \\
& R^{4}: \text { If } x(t) \text { is } F_{1}^{2} \text { and } \dot{x}(t) \text { is } F_{2}^{2}, \text { then } u(t)=r_{4}(t) \text {. }
\end{aligned}
$$

Accordingly, the firing-strength of each rule is

$$
\begin{aligned}
& \alpha_{1}(X(t))=F_{1}^{1}(x) \cdot F_{2}^{1}(\dot{x}) \\
& \alpha_{2}(X(t))=F_{1}^{1}(x) \cdot F_{2}^{2}(\dot{x}) \\
& \alpha_{3}(X(t))=F_{1}^{2}(x) \cdot F_{2}^{1}(\dot{x}) \\
& \alpha_{4}(X(t))=F_{1}^{2}(x) \cdot F_{2}^{2}(\dot{x})
\end{aligned}
$$

where the normalized firing-strength of the $i$ th rule is

$$
h_{i}(X(t))=\alpha_{i} / \sum_{i=1}^{4} \alpha_{i}
$$

Now, let the penalty matrices be set as $L=I_{2}$ and $Q=I_{2}$. Then, the designed finite-horizon optimal controller according to (13) and (14) is

$$
\begin{aligned}
u^{*}(t) & =\sum_{1}^{4} h_{i}(X(t)) r_{i}^{*}(t) \\
& \text { with } r_{i}^{*}(t)=-B_{i}^{T} \pi^{i}\left(t, I_{2}, t_{1}\right) X^{*}(t), \quad i=1, \cdots, 4
\end{aligned}
$$

where $\pi^{i}\left(t, I_{2}, t_{1}\right)$ is the symmetric positive-semidefinite solution of the matrix Riccati differential equation in (12). Since $\pi^{i}\left(t, I_{2}, t_{1}\right)$ always exists and the above controller stabilizes the subsystem at any $t \in\left[t_{0}, t_{1}\right]$, the entire feedback system is exponentially stable, which can be observed from the state response of the closed-loop fuzzy system at different initial conditions in Fig. 1.

Since the fuzzy subsystem is time-invariant and well-behaved; i.e., the subsystem is c.c. and c.o. $\left(\operatorname{rank}\left[A_{i} A_{i} B_{i}\right]=2\right.$ and $\operatorname{rank}\left[C^{T} A_{i}^{T} C^{T}\right]^{T}=2$ for $\left.i=1, \cdots, 4\right)$, there exists a unique symmetric positive semidefinite solution, $\pi_{\infty}^{i}, i=$ $1, \cdots, 4$, of the S.S.R.E. in (16)

$$
\begin{array}{ll}
\pi_{\infty}^{1}=\left[\begin{array}{ll}
1.7206 & 0.9802 \\
0.9802 & 1.7209
\end{array}\right], & \pi_{\infty}^{2}=\left[\begin{array}{ll}
1.5102 & 0.9802 \\
0.9802 & 1.7311
\end{array}\right] \\
\pi_{\infty}^{3}=\left[\begin{array}{ll}
1.2635 & 0.2982 \\
0.2982 & 2.3068
\end{array}\right], & \pi_{\infty}^{4}=\left[\begin{array}{ll}
1.0584 & 0.2982 \\
0.2982 & 1.9994
\end{array}\right]
\end{array}
$$

and the asymptotically optimal controller is

$u^{*}(t)=\sum_{1}^{4} h_{i}\left(X^{*}(t)\right) r_{i}^{*}(t), \quad$ with $r_{i}^{*}(t)=-B_{i}^{T} \pi_{\infty}^{i} X^{*}(t)$. 

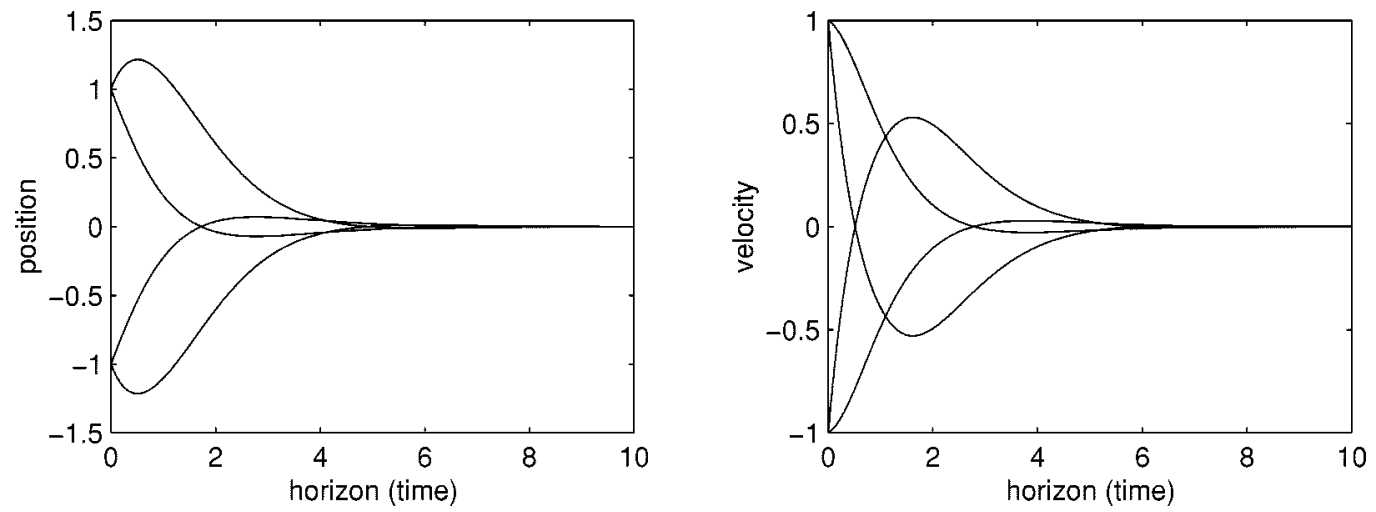

Fig. 1. The state responses of the continuous-time fuzzy system with the designed optimal controller in the finite-horizon quadratic optimal control problem of Section V-A at the four initial conditions: $X(0)=(-1,-1)^{T},(-1,1)^{T},(1,-1)^{T}$, and $(1,1)^{T}$.
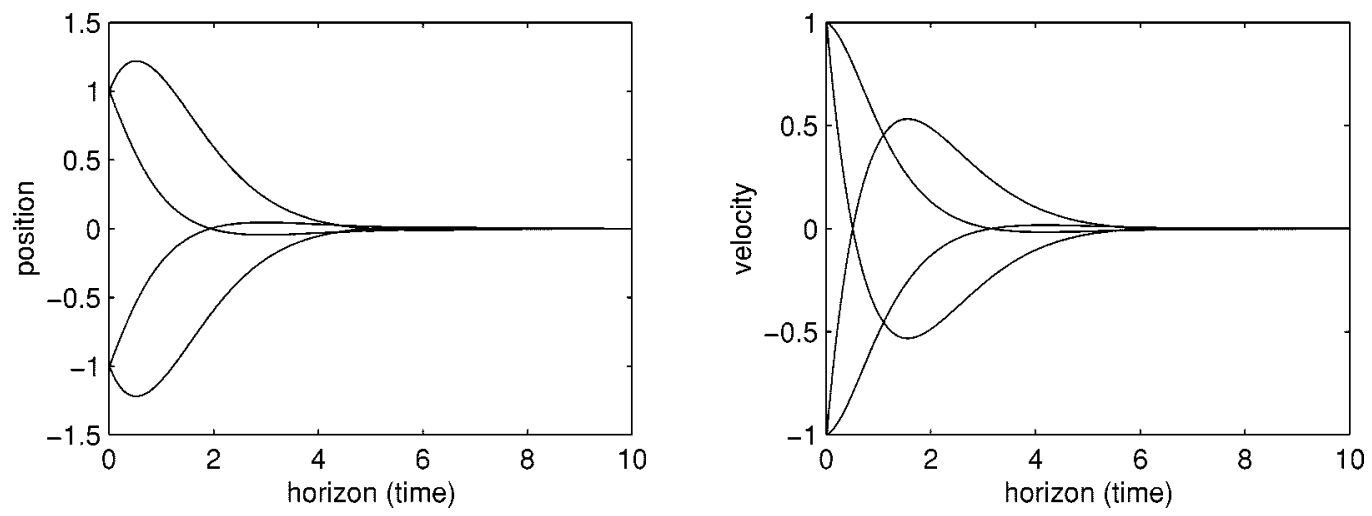

Fig. 2. The state responses of the continuous-time fuzzy system with the designed optimal controller in the infinite-horizon quadratic optimal control problem of Section V-A at the four initial conditions: $X(0)=(-1,-1)^{T},(-1,1)^{T},(1,-1)^{T}$, and $(1,1)^{T}$.

The optimal feedback fuzzy system, (30), is exponentially stable, the total energy of system output is finite, and moreover, this optimal controller can stabilize the fuzzy system to any prescribed degree of stability and generates a closed-loop fuzzy system with an infinite gain margin. Fig. 2 illustrates the position and velocity responses of the closed-loop fuzzy system in different initial conditions. From the simulation results, we find the designed optimal fuzzy controller can quickly push the system from various initial states to and stay at the desired state in both cases of finite and infinite horizons.

\section{B. Discrete-Time System}

Tanaka and Sano [29] described a computer simulated trucktrailer with the mathematical model

$$
\begin{aligned}
& x_{1}(t+1)=\left(1-v \cdot t^{\prime} / L^{\prime}\right) x_{1}(t)+v \cdot t^{\prime} / l \cdot u(t) \\
& x_{2}(t+1)=x_{2}(t)+v \cdot t^{\prime} / L^{\prime} \cdot x_{1}(t) \\
& x_{3}(t+1)=x_{3}(t)+v \cdot t^{\prime} \cdot \sin \left(x_{2}(t)+v \cdot t^{\prime} / 2 L^{\prime} \cdot x_{1}(t)\right)
\end{aligned}
$$

where $l$ is the length of truck, $L^{\prime}$ is the length of trailer, $t^{\prime}$ is the sampling time, and $v$ is the constant speed of the backward movement. Then, they used the following fuzzy model to represent the truck-trailer system:

$$
\begin{aligned}
& R^{1}: \text { If } z(t) \equiv x_{2}(t)+v \cdot t^{\prime} /\left\{2 L^{\prime}\right\} \cdot x_{1}(t) \text { is about } 0, \\
& \quad \text { then } X(t+1)=A_{1} X(t)+B_{1} u(t)
\end{aligned}
$$

$$
\begin{aligned}
& R^{2}: \text { If } z(t) \equiv x_{2}(t)+v \cdot t^{\prime} /\left\{2 L^{\prime}\right\} \cdot x_{1}(t) \text { is about } \pi \text { or }-\pi \\
& \quad \text { then } X(t+1)=A_{2} X(t)+B_{2} u(t)
\end{aligned}
$$

and the system output is $Y(t)=C X(t)$ with $C=I_{3}, l=2.8$, $L^{\prime}=5.5, v=-1.0, t^{\prime}=2.0$ and

$$
X(t)=\left[x_{1}(t) x_{2}(t) x_{3}(t)\right]^{T}
$$

where

$$
\begin{aligned}
& A_{1}=\left[\begin{array}{ccc}
1.3636 & 0 & 0 \\
-0.3636 & 1.0 & 0 \\
0.0120 & -2.0 & 1.0
\end{array}\right] \\
& A_{2}=\left[\begin{array}{ccc}
1.3636 & 0 & 0 \\
-0.3636 & 1.0 & 0 \\
0 & -0.0064 & 1.0
\end{array}\right] \\
& B_{1}=B_{2}=\left[\begin{array}{c}
-0.7143 \\
0 \\
0
\end{array}\right] .
\end{aligned}
$$

We further assume our fuzzy controller is

$$
\begin{aligned}
& R^{1}: \text { If } z(t) \equiv x_{2}(t)+v \cdot t^{\prime} /\left\{2 L^{\prime}\right\} \cdot x_{1}(t) \text { is about } 0 \\
& \quad \text { then } u(t)=r_{1}(t) \\
& R^{2}: \text { If } z(t) \equiv x_{2}(t)+v \cdot t^{\prime} /\left\{2 L^{\prime}\right\} \cdot x_{1}(t) \text { is about } \pi \text { or }-\pi \\
& \quad \text { then } u(t)=r_{2}(t) .
\end{aligned}
$$



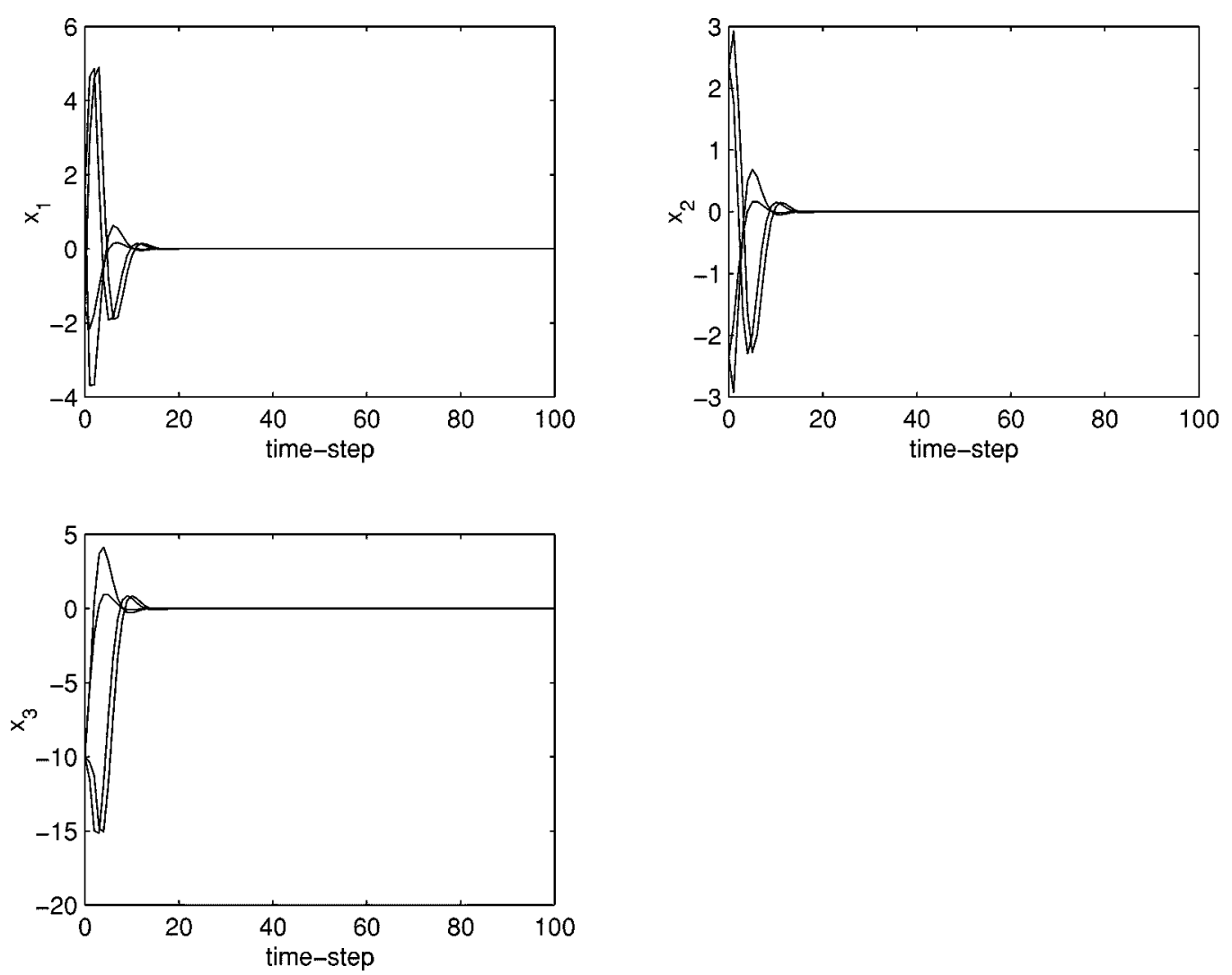

Fig. 3. The state responses of the discrete-time fuzzy system with the designed optimal controller in the finite-horizon quadratic optimal control problem of Section V-B at the four initial conditions: $X(0)=(-\pi / 2,-3 \pi / 4,-10)^{T},(-\pi / 2,3 \pi / 4,-10)^{T},(\pi / 2,-3 \pi / 4,-10)^{T}$, and $(\pi / 2,3 \pi / 4,-10)^{T}$.

With the chosen membership functions, the normalized firingstrength is

$$
\begin{aligned}
\alpha_{1}(t)= & (1-1 /(1+\exp (-3(z(t)-\pi / 2)))) \\
& \cdot(1 / 1+\exp (-3(z(t)+\pi / 2)))) \\
\alpha_{2}(t)= & 1-\alpha_{1}(t) .
\end{aligned}
$$

Given the penalty matrices $L=I_{3}$ and $Q=I_{3}$, the designed finite-horizon optimal controller according to (21) and (22) is

$$
\begin{aligned}
u^{*}(t) & =\sum_{i=1}^{2} h_{i}\left(X^{*}(t)\right) r_{i}^{*}(t), \quad \text { with } \\
r_{i}^{*}(t) & =-\left[I_{3}+B_{i}^{T} \pi^{i}(t+1) B_{i}\right]^{-1} B_{i}^{T} \pi^{i}(t+1) A_{i} X^{*}(t) \\
i & =1,2
\end{aligned}
$$

where $\pi^{i}(t)$ is the symmetric positive-semidefinite solution of the matrix Riccati difference equation in (20) or (24).

The original subsystem is unstable, since

$$
\boldsymbol{\sigma}\left(A_{i}\right)=\{1,1,1.36\}, \quad i=1,2 \text {. }
$$

However, $\operatorname{rank}\left[\lambda I_{3}-A_{i} B_{i}\right]=3$ and $\operatorname{rank}\left[\lambda I_{3}-A_{i} C\right]=3$, for all $\lambda$ in the intersection of the spectrum of $A_{i}$ and the complement of open unit disk, i.e., $\forall \lambda \in \boldsymbol{\sigma}\left(A_{i}\right) \cap D(0,1)^{c}$; accordingly, $\left(A_{i}, B_{i}\right)$ is stabilizable and $\left(A_{i}, C\right)$ is detectable, $i=$
1,2 . Hence, all the discrete-time subsystems are still well-behaved. The unique symmetric positive-semidefinite solution of the S.S.R.E. in (25) or (26) is

$$
\begin{aligned}
\pi^{1}(\infty) & =\left[\begin{array}{ccc}
13.1413 & -27.4324 & 3.8860 \\
-27.4324 & 90.2599 & -15.7378 \\
3.8860 & -15.7378 & 4.5296
\end{array}\right] \\
\pi^{2}(\infty) & =\left[\begin{array}{ccc}
4.9768 & -2.7158 & 2.6338 \\
-2.7158 & 5.6080 & -5.4833 \\
2.6338 & -5.4833 & 162.9712
\end{array}\right]
\end{aligned}
$$

and the local optimal fuzzy control law is

$$
\begin{aligned}
& u^{*}(t)=\sum_{i=1}^{2} h_{i}\left(X^{*}(t) r_{i}^{*}(t), \quad\right. \text { with } \\
& r_{i}^{*}(t)=-\left[I_{3}+B_{i}^{T} \pi^{i}(\infty) B_{i}\right]^{-1} B_{i}^{T} \pi^{i}(\infty) A_{i} X^{*}(t), \\
& t=t_{0}, \cdots, N-1 .
\end{aligned}
$$

By the way, the designed optimal controller can stabilize the local system.

However, there is no straightforward relationship between the stability of subsystems and that of the entire system for the discrete-time system. We may adopt Lyapunov's direct method [8] to perform the stability analysis of overall feedback system. Figs. 3 and 4 show the tracking results in various poor initial conditions for finite-horizon and infinite-horizon optimal control problems, respectively. Obviously, a perfect, fast tracking is achievable even when the variation of the initial states occurs. 

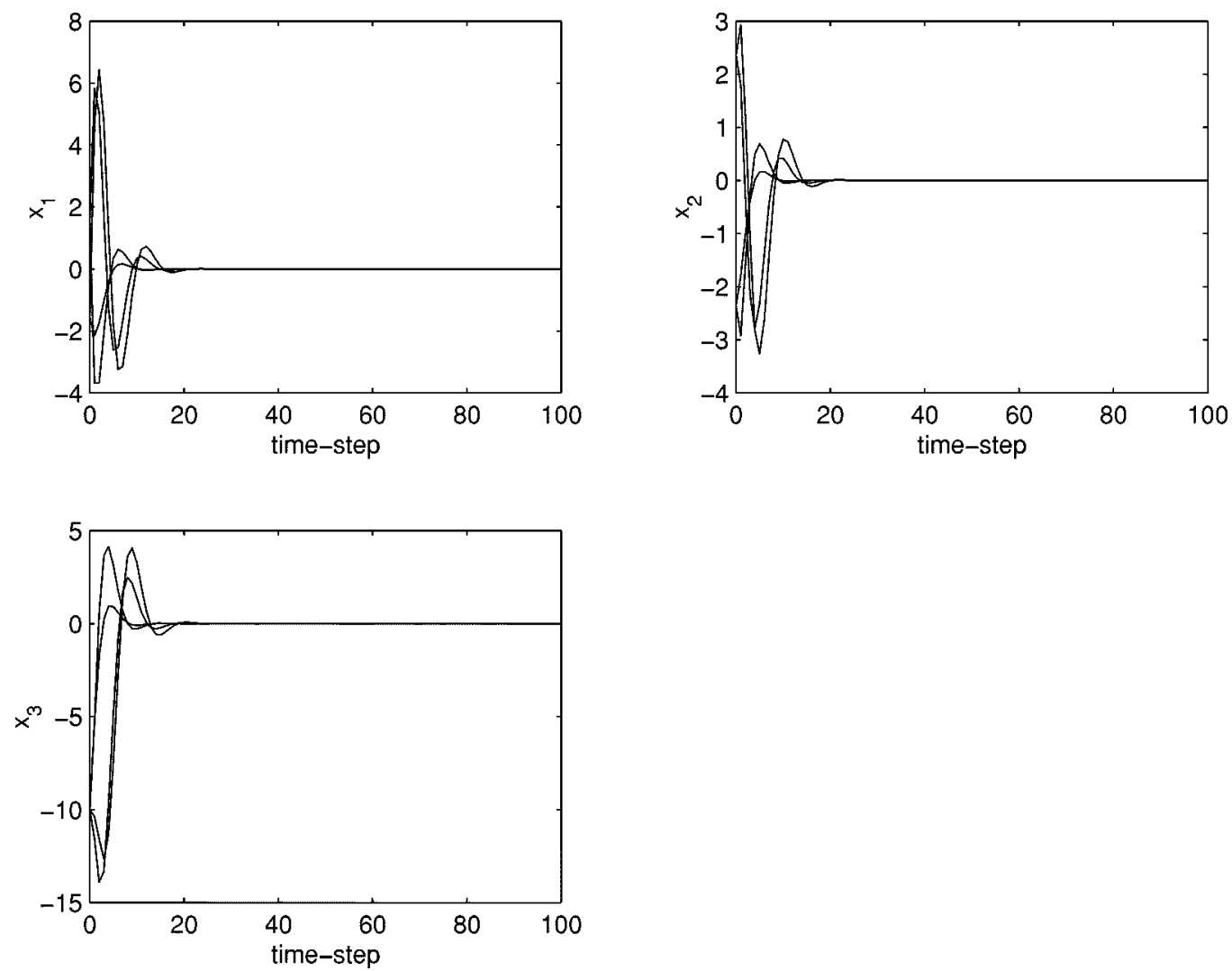

Fig. 4. The state responses of the discrete-time fuzzy system with the designed optimal controller in the infinite-horizon quadratic optimal control problem of Section V-B at the four initial conditions: $X(0)=(-\pi / 2,-3 \pi / 4,-10)^{T},(-\pi / 2,3 \pi / 4,-10)^{T},(\pi / 2,-3 \pi / 4,-10)^{T}$, and $(\pi / 2,3 \pi / 4,-10)^{T}$.

\section{CONCLUSION}

A sufficient condition for global optimal fuzzy control was proposed in this paper. This condition shows that "blending" optimal local fuzzy controllers can achieve global optimal effect. Based on this observation, the design scheme of finite-horizon global optimal fuzzy controllers in continuous-time system as well as in discrete-time system were derived. In the case of time-invariant and well-behaved fuzzy systems, the design scheme of infinite-horizon global optimal fuzzy controllers for both the continuous-time and discrete-time systems were also obtained. Several fascinating characteristics have been shown to exist in the closed-loop continuous-time fuzzy system for the infinite-horizon optimal control problem. First, we have shown that the stability of the entire closed-loop fuzzy system can be guaranteed if the simple completely controllable and completely observable criteria hold for the fuzzy subsystems. Furthermore, under this situation, the closed-loop fuzzy system has freedom in the choice of the degree of stability and gain margin, meaning that the designed optimal fuzzy controller can stabilize the fuzzy system to any desired degree of stability and the resultant closed-loop fuzzy system possesses an infinite gain margin. Simulation results have manifested that all the designed optimal fuzzy controllers can effectively drive the fuzzy system to the target points in a short time.

\section{APPENDIX}

We describe here the related optimal control concepts and results adopted in this paper. We start with finite-horizon optimal control problem in continuous-time systems, and then, the infinite-horizon issue. Next, we tackle these two optimal control problems in discrete-time systems, since it is difficult to get complete bibliography on this issue, and even, the mathematical statements for the discrete-time systems are always diverse.

\section{A. Continuous-Time Systems}

We consider here a dynamical system represented by the following equation:

$$
\dot{X}(t)=A(t) X(t)+B(t) u(t), \quad \text { for } t \in \Re_{+}
$$

where the state $X(t) \in \Re^{n}$, the input $u(t) \in \Re^{m}$, and $A(\cdot)$, $B(\cdot)$ are matrix-valued functions on $\Re_{+}$of class PC. The general LQ problem is to find an optimal control law $u^{*}(\cdot)$ to minimize the performance index $J(u(\cdot))$ in (3). The results shown in [24], [27], [28], [30]-[32] are summarized as follows.

Proposition 4 [27], [31]: Let $A(t), B(t), L(t)=L^{T}(t) \geq 0$, $Q=Q^{T} \geq 0$ be given matrices. Suppose the matrix Riccati equation

$\dot{K}(t)=-A^{T}(t) K(t)-K(t) A(t)+K(t) B(t) B^{T}(t) K(t)-L(t)$

with $K\left(t_{1}\right)=Q$ has a solution $\pi\left(t, Q, t_{1}\right)$ on the interval $\left[t_{0}, t_{1}\right]$. Then, there exists a controller $u^{*}(\cdot)$ which minimizes $J(u(\cdot))$ in (3) for the system in (49) with $X\left(t_{0}\right)=X_{0}$. And, the optimal control law is

$$
u^{*}(t)=-B^{T}(t) \pi\left(t, Q, t_{1}\right) X^{*}(t) .
$$


Furthermore, the minimum value of $J(u(\cdot))$ is $X_{0}^{T} \pi\left(t_{0}, Q, t_{1}\right) X_{0}$.

Next, when the horizon $t_{1}$ extends to infinity, it belongs to the infinite-horizon optimal control problem. In this case, we, moreover, assume $L(t)=C^{T}(t) C(t)$ or $C(t)=L^{1 / 2}(t)$. In other words, the system output is $Y(t)=C(t) X(t)$. Then, the performance index is finite if the system is completely controllable and the stability of the feedback system is guaranteed if the system is completely observable. For the time-invariant system, a more attractive characteristic listed below is elicited. Here, we use $\pi_{\infty}$ to denote the limit value of the solution of the aforementioned matrix Riccati equation, i.e.,

$$
\pi_{\infty} \triangleq \lim _{t_{1} \rightarrow \infty} \pi\left(t_{0}, Q, t_{1}\right)
$$

Proposition 5 [30]: If $L$ is positive definite and $(A, B)$ is c.c., then $\pi_{\infty}$ exists and satisfies the S.S.R.E.

$$
A^{T} K+K A-K B B^{T} K+L=0 .
$$

Moreover, $\pi_{\infty}$ is positive-definite and symmetric.

Proposition 6 [24], [28]: If $(A, B)$ is c.c., $(A, C)$ is c.o., and $\pi_{\infty}$ is the positive-definite solution of the S.S.R.E. in Proposition 5 , then

1) $u^{*}(t)=-B^{T} \pi_{\infty} X(t)$ is the optimal control law which minimizes $J(u(\cdot)$ in $(18)$;

2) all the solutions of the feedback system

$$
\dot{X}(t)=\left[A-B B^{T} \pi_{\infty}\right] X(t)
$$

tend to 0 as $t \rightarrow \infty$; that is, it is asymptotically stable.

Proposition 7 [31], [32]: If $(A, B)$ is c.c. and $(A, C)$ is c.o., then the system can be stabilized to any desired degree; i.e., all the eigenvalues of the feedback system have real parts smaller than $-\alpha$, for all $\alpha \in \Re_{+}^{\circ}$.

\section{B. Discrete-Time Systems}

This section discusses how to obtain the optimal control law for the discrete-time system. We shall first introduce the issue of the finite-horizon optimal control problem, and then the infinitehorizon optimal control problem. Assume our system is

$$
\begin{aligned}
X(t+1)=A(t) X(t) & +B(t) u(t), \\
X(0) & =X_{0} \in \Re^{n}, t=t_{0}, \cdots, N-1 .
\end{aligned}
$$

The finite-horizon optimal control problem is to search an optimal control law $u^{*}(\cdot)$ to minimize $J(u(\cdot))$ in (4).

Proposition 8: Let $A(t), B(t), L(t)=L^{T}(t) \geq 0$, $Q=Q^{T} \geq 0$ be given matrices. Suppose the matrix Riccati equation

$$
\begin{array}{r}
V(t)=-A^{T}(t) V(t+1) B(t)\left[I_{n}+B^{T}(t) V(t+1) B(t)\right]^{-1} \\
\times B^{T}(t) V(t+1) A(t) L(t)+A^{T}(t) V(t+1) A(t) \\
t=t_{0}, \cdots, N-1
\end{array}
$$

with $V(N)=Q$, has a solution $\pi(t), t=t_{0}, \cdots, N-1$, then there exists an optimal control law $u^{*}(t)$ which minimizes $J(u(\cdot))$ in (4) for the system in (53) with $X(0)=X_{0}$. The optimal control law is

$u^{*}(t)=-\left[I_{n}+B^{T}(t) \pi(t+1) B(t)\right]^{-1} B^{T}(t) \pi(t+1) A(t) X(t)$ and the corresponding optimal trajectory is

$$
X^{*}(t+1)=\left[I_{n}+B(t) B^{T}(t) \pi(t+1)\right]^{-1} A(t) X^{*}(t) .
$$

Moreover, the minimum value of $J(u(\cdot))$ is $X_{0}^{T} \pi(0) X_{0}$, and, via some standard matrix manipulations, the matrix Riccati difference equation in (54) can be rewritten as

$$
V(t)=L(t)+A^{T}(t) V(t+1)\left[I_{n}+B(t) B^{T}(t) V(t+1)\right]^{-1} A(t)
$$

Proof: 1) Define

$$
\begin{aligned}
\Phi_{N}(X(t)) \triangleq \min _{\left\{u\left(t^{\prime}\right)\right\}}\{ & \sum_{t^{\prime}=t}^{N-1}\left[X^{T}\left(t^{\prime}\right) L\left(t^{\prime}\right) X\left(t^{\prime}\right)+u^{T}\left(t^{\prime}\right) u\left(t^{\prime}\right)\right] \\
& \left.+X^{T}(N) Q X(N)\right\} .
\end{aligned}
$$

By the principle of optimality in dynamic programming in [33], [34], the above equation can be rewritten as

$\Phi_{N}(X(t))=\min _{u(t)}\left[X^{T}(t) L(t) X(t)+u^{T}(t) u(t)+\Phi_{N}(\mathrm{x}(t+1))\right]$.

We notice that $\Phi_{N}(X(N))=X^{T}(N) Q X(N)$. Therefore, want to look for a solution of the form

$$
\Phi_{N}(X(t))=X^{T}(t) V(t) X(t)
$$

with $V(N)=Q$, where $V(t)$ is the introduced time step variable. Hence, we have

$$
X^{T}(t) V(t) X(t)=\min _{u(t)}\left\{X^{T}(t) L(t) X(t)+\Psi(u(t))\right\}
$$

where

$$
\begin{aligned}
\Psi(u(t))= & u^{T}(t) u(t)+[A(t) X(t)+B(t) u(t)]^{T} V(t+1) \\
& \times[A(t) X(t)+B(t) u(t)] .
\end{aligned}
$$

Then, we perform the minimization of $\Psi(u(t))$ with respect to $u(t)$

$$
\begin{aligned}
\nabla_{u(t)} \Psi(u(t))= & 2 u(t)+2 B^{T}(t) V(t+1) \\
& \times[A(t) X(t)+B(t) u(t)]=0 .
\end{aligned}
$$

Accordingly, we can obtain

$$
\begin{aligned}
u^{*}(t)= & -B^{T}(t) V(t+1)\left[A(t) X(t)+B(t) u^{*}(t)\right] \\
u^{*}(t)= & -\left[I_{n}+B^{T}(t) V(t+1) B(t)\right]^{-1} \\
& \times B^{T}(t) V(t+1) A(t) X(t) .
\end{aligned}
$$

Substituting (57) and (58) into (56), we have

$$
\begin{aligned}
X_{t}^{T} V_{t} X_{t}= & X_{t}^{T} L_{t} X_{t}+u_{t}^{*^{T}} u_{t}^{*} \\
& +\left(A_{t} X_{t}+B_{t} u_{t}^{*}\right)^{T} V_{t+1}\left(A_{t} X_{t}+B_{t} u_{t}^{*}\right) \\
= & X_{t}^{T} L_{t} X_{t}+X_{t}^{T} A_{t} V_{t+1}\left(A_{t} X_{t}+B_{t} u_{t}^{*}\right)
\end{aligned}
$$


by denoting time dependence as a lower index. Furthermore, via (58), we have

$$
\begin{aligned}
X_{t}^{T} V_{t} X_{t}= & X_{t}^{T} L_{t} X_{t}+X_{t}^{T} A_{t} V_{t+1} A_{t} X_{t} \\
& -X_{t}^{T} A_{t} V_{t+1} B_{t}\left[I_{n}+B_{t}^{T} V_{t+1} B_{t}\right]^{-1} \\
& \times B_{t}^{T} V_{t+1} A_{t} X_{t} .
\end{aligned}
$$

Hence, (54) holds. Now, we shall show that (54) and (55) are equivalent via the matrix manipulations as below. By omitting the time-dependence index at time-step $t$ for notation simplification, we have

$$
\begin{aligned}
V & =L+A^{T} V_{t+1} A-A^{T} V_{t+1} B\left[I_{n}+B^{T} V_{t+1} B\right]^{-1} B^{T} V_{t+1} A \\
& =L+A^{T} V_{t+1}\left(I_{n}-B\left[I_{n}+B^{T} V_{t+1} B\right]^{-1} B^{T} V_{t+1}\right) A
\end{aligned}
$$

which is equivalent to (55) since

$$
\begin{aligned}
& {\left[I_{n}+B B^{T} V_{t+1}\right] \cdot\left(I_{n}-B\left[I_{n}+B^{T} V_{t+1} B\right]^{-1} B^{T} V_{t+1}\right)=I_{n}} \\
& \left(I_{n}-B\left[I_{n}+B^{T} V_{t+1} B\right]^{-1} B^{T} V_{t+1}\right) \cdot\left[I_{n}+B B^{T} V_{t+1}\right]=I_{n}
\end{aligned}
$$

Then, substituting (55) into $X^{*}(t+1)=A(t) X^{*}(t)+$ $B(t) u^{*}(t)$, we obtain

$$
\begin{aligned}
X^{*}(t+1)= & \left\{I_{n}-B(t)\left[I_{n}+B^{T}(t) V(t+1) B(t)\right]^{-1}\right. \\
& \left.\times B^{T}(t) V(t+1)\right\} A(t) X(t) \\
= & {\left[I_{n}+B(t) B^{T}(t) V(t+1)\right]^{-1} A(t) X(t) . }
\end{aligned}
$$

This completes the proof.

Now, we turn to the infinite-horizon optimal control problem with time-invariant system and use $V(\infty)$ to denote the limit value of the matrix Riccati difference equation in (54) or (55), i.e., $V(\infty) \triangleq \lim _{N \rightarrow \infty} V(t)$.

Proposition 9 [24]: Let $A, B, L=C^{T} C$ be given matrices. If $(A, B)$ is stabilizable and $(C, A)$ is detectable, then

1) there exists a unique symmetric positive semidefinite solution $\pi(\infty)$ of the S.S.R.E.

$$
V(\infty)=L+A^{T} V(\infty)\left[I_{n}+B B^{T} V(\infty)\right]^{-1} A
$$

which can be rewritten as

$$
\begin{aligned}
V(\infty)= & L+A^{T} V(\infty) A \\
& -A^{T} V(\infty) B\left[I_{n}+B^{T} V(\infty) B\right]^{-1} B^{T} V(\infty) A
\end{aligned}
$$

2) the asymptotically optimal control law is

$$
u^{*}(t)=-\left[I_{n}+B^{T} \pi(\infty) B\right]^{-1} B^{T} \pi(\infty) A X(t)
$$

which minimizes $J(u(\cdot))$ in (28), and the minimum value of $J(u(\cdot))$ is $X_{0}^{T} \pi(\infty) X_{0}$;

3 ) the closed-loop feedback system is asymptotically and exponentially stable.

\section{REFERENCES}

[1] H. Wang, K. Tanaka, and M. Griffin, "Parallel distributed compensation of nonlinear systems by Takagi and Sugeno's fuzzy model," in Proc. FUZZ-IEEE'95, 1995, pp. 531-538.

[2] H. O. Wang, K. Tanaka, and M. F. Griffin, "An approach to fuzzy control of nonlinear system: Stability and design issues," IEEE Trans. Fuzzy Syst., vol. 4, pp. 14-23, Feb. 1996.

[3] K. Tanaka, T. Ikeda, and H. O. Wang, "Robust stabilization of a class of uncertain nonlinear systems via fuzzy control: Quadratic stabilizability, $H^{\infty}$ control theory and linear matrix inequalities," IEEE Trans. Fuzzy Syst., vol. 4, pp. 1-13, Jan. 1996.

[4] H. O. Wang and K. Tanaka, "An LMI-based stable fuzzy control of nonlinear systems and its application to control of chaos," in Proc. FUZZIEEE'96, vol. 2, 1996, pp. 1433-1438.

[5] K. Tanaka and T. Kosaki, "Design of a stable fuzzy controller for an articulated vehicle," IEEE Trans. Syst., Man, Cybern. B, vol. 27, pp. 552-558, June 1997.

[6] K. Tanaka, T. Taniguchi, and H. O. Wang, "Model-based fuzzy control of TORA system: Fuzzy regulator and fuzzy observer design via LMI's that represent decay rate, disturbance rejection, robustness, optimality," in Proc.FUZZ-IEEE'98, 1998, pp. 313-318.

[7] K. Tanaka, T. Kosaki, and H. O. Wang, "Fuzzy control of a mobile robot with multiple trailers-stability analysis and parallel distributed compensation," in Proc. IEEE Intell. Contr., 1996, pp. 259-264.

[8] K. Tanaka and M. Sugeno, "Stability analysis and design of fuzzy control systems," Fuzzy Sets Syst., vol. 45, pp. 135-156, 1992.

[9] X. J. Ma, Z. Q. Sun, and Y. Y. He, "Analysis and design of fuzzy controller and fuzzy observer," IEEE Trans. Fuzzy Syst., vol. 6, pp. 41-51, Feb. 1998.

[10] S. G. Cao, N. W. Rees, and G. Feng, "Analysis and design for a class of complex control systems-Part I: Fuzzy modeling and identification," Automatica, vol. 33, no. 6, pp. 1017-1028, 1997.

[11] _ "Analysis and design for a class of complex control systems-Part II: Fuzzy controller design,” Automatica, vol. 33, no. 6, pp. 1029-1039, 1997.

[12] L. X. Wang, "Stable and optimal fuzzy control of linear systems," IEEE Trans. Fuzzy Syst., vol. 6, pp. 137-143, Feb. 1998.

[13] K. Tanaka, T. Ikeda, and H. O. Wang, "Design of fuzzy control systems based on relaxed LMI stability condition," in IEEE Conf. Decision Contr, vol. 1, 1996, pp. 598-603.

[14] _ " "An LMI approach to fuzzy controller designs based on relaxed stability conditions," in Proc. FUZZ-IEEE'97, vol. 1, 1997, pp. 171-176.

[15] K. Tanaka and M. Sano, "Trajectory stabilization of a model car via fuzzy control," Fuzzy Sets Syst., vol. 70, pp. 155-170, 1995.

[16] - "Frequency shaping for fuzzy control systems with unkown nonlinear plants by a learning methods of neural network," Fuzzy Sets Syst., vol. 71, pp. 71-84, 1995.

[17] H. K. Lam, F. H. F. Leung, and P. K. S. Tam, "Stable and robust fuzzy control for uncertain nonlinear systems based on a grid-point approach," in Proc. FUZZ-IEEE'97, vol. 1, 1997, pp. 281-285.

[18] H. J. Kang and C. Kwon, " $L^{\infty}$ robust stability analysis for the fuzzy feedback linearization regulator," in Proc. FUZZ-IEEE'97, vol. 1, 1997, pp. 277-280.

[19] K. S. Ray and D. D. Maunder, "Application of the circle criteria for stability analysis of linear SISO and MIMO systems associated with fuzzy logic controllers," IEEE Trans. Syst., Man, Cybern., vol. 2, pp. 345-349, Mar./Apr. 1984.

[20] L. X. Wang, "A supervisory controller for fuzzy control systems that guarantees stability," in Proc. FUZZ-IEEE'94, Orlando, FL, 1994, pp. $1035-1039$.

[21] K. Tanaka, T. Taniguchi, and H. O. Wang, "Fuzzy control based on quadratic performance function," in 37th IEEE Conf. Decision Contr., Tampa, FL, 1998, pp. 2914-2919.

[22] — " "Model-based fuzzy control of TORA system: Fuzzy regulator and fuzzy observer design via LMI's that reprensent decay rate, disturbance rejection, robustness, optimality," in Proc. FUZZ-IEEE'98, Alaska, 1998, pp. 313-318.

[23] C. T. Lin and C. S. G. Lee, "Reinforcement structure/parameter learning for neural-network-based fuzzy logic control systems," IEEE Trans. Fuzzy Syst., vol. 2, pp. 46-63, Feb. 1994.

[24] B. D. O. Anderson and J. B. Moore, Optimal Control: Linear Quadratic Methods. Englewood Cliffs, NJ: Prentice-Hall, 1990

[25] M. Vidyasagar, Nonlinear Systems Analysis. Englewood Cliffs, NJ: Prentice-Hall, 1993.

[26] P. R. Halmos, A Hilbert Space Problem Book. New York: SpringerVerlag, 1982 
[27] F. M. Callier and C. A. Desoer, Linear System Theory. New York: Springer-Verlag, 1991.

[28] Lecture Notes. Prof. P. K. C. Wang, Course EE240B, Univ. California, Los Angeles)

[29] S. G. Cao, N. W. Rees, and G. Feng, "Lyapunov-like stability theorems for discrete-time fuzzy control systems," Int. J. Syst. Sci., vol. 28, no. 3, pp. 297-308, 1997.

[30] R. E. Kalman, "When is a linear control system optimal?," Trans. ASME Ser. D: J. Basic Eng., vol. 86, pp. 1-10, Mar. 1964.

[31] B. D. O. Anderson, Linear Optimal Control. Englewood Cliffs, NJ: Prentice-Hall, 1971.

[32] B. D. O. Anderson and J. B. Moore, "Linear system optimization with prescribed degree of stability," Proc. Inst. Elect. Eng., vol. 116, no. 12, pp. 2083-2087, 1969.

[33] S. E. Dreyfus, Dynamic Programming and the Calculus of Variations. New York: Academic, 1965.

[34] R. E. Bellman and S. E. Dreyfus, Applied Dynamic Programming. Princeton, NJ: Princeton Univ. Press, 1962.

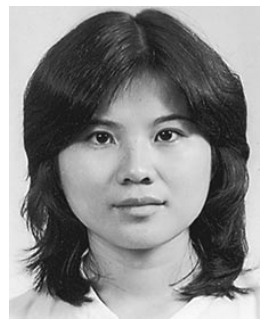

Shinq-Jen $\mathbf{W u}$ received the B.S. degree in chemical engineering from the National Taiwan University, Taipei, Taiwan, R.O.C., in 1986, the M.S. degree in chemical engineering from the National Tsing-Hua University, Hsinchu, Taiwan, R.O.C., in 1989, and the M.S. degree in electrical engineering from the University of California, Los Angeles, CA, in 1994 She is currently working toward the Ph.D. degree in electrical engineering at the National Chiao-Tung University, Hsinchu, Taiwan, R.O.C.

From September 1989 to July 1990, she was with the Laboratory for Simulation and Control Technology of the Chemical Engineering Division of Industrial Technology Research Institute, Hsinchu, Taiwan, R.O.C. Since then, until 1991, she was with the Chemical Engineering Department, Kao-Yuan Junior College of Technology and Commerce at Kaohsiung, Taiwan, R.O.C. From 1995 to 1996 she was an Engineer at the Integration Engineering Department, Macronix International Co., Ltd, Hsinchu, Taiwan, R.O.C. Her research interests include thermodynamics, transport phenomena, process control and design, especially in VLSI and the petroleum industry, system and control theory, especially in optimal control, filtering theory, fuzzy system theory, and optimal fuzzy controller and tracker design.

Ms. Wu is a member of Phi-Tau-Phi scholastic honor society.

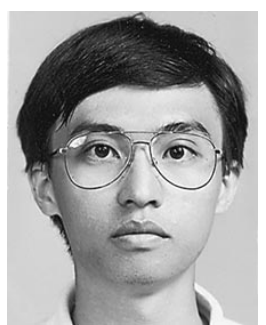

Chin-Teng Lin (S'88-M'91-SM'95) received the B.S. degree in control engineering from the National Chiao-Tung University, Hsinchu, Taiwan, R.O.C., in 1986, and the M.S.E.E. and Ph.D. degrees in electrical engineering from Purdue University, West Lafayette, IN, in 1989 and 1992, respectively.

Since August 1992, he has been with the College of Electrical Engineering and Computer Science, National Chiao-Tung University, Hsinchu, Taiwan, R.O.C., where he is currently a Professor of Electrical and Control Engineering. He has also served as the Deputy Dean of the Research and Development Office of the National Chiao-Tung University since 1998. His current research interests are fuzzy systems, neural networks, intelligent control, human-machine interface, and video and audio processing. He is the coauthor of Neural Fuzzy Systems-A Neuro-Fuzzy Synergism to Intelligent Systems (Englewood Cliffs, NJ: Prentice-Hall), and the author of Neural Fuzzy Control Systems with Structure and Parameter Learning (Singapore: World Scientific). He has published over 45 journal papers in the areas of neural networks and fuzzy systems.

Dr. Lin is a member of Tau Beta Pi and Eta Kappa Nu. He is also a member of the IEEE Computer Society, the IEEE Robotics and Automation Society, and the IEEE Systems, Man, Cybernetics Society. He has been the Executive Council Member of the Chinese Fuzzy System Association (CFSA) since 1995, and the Supervisor of the Chinese Automation Association since 1998. He was the Vice Chairman of IEEE Robotics and Automation Taipei Chapter in 1996 and 1997. He won the Outstanding Research Award granted by National Science Council (NSC), Taiwan, in 1997 and 1999, and the Outstanding Electrical Engineering Professor Award granted by the Chinese Institute of Electrical Engineering (CIEE) in 1997. 